\title{
LABORATORY RECTIFYING STILLS OF GLASS 12
}

\author{
By Johannes H. Bruun ${ }^{3}$ and Sylvester T. Schicktanz ${ }^{3}$
}

\section{ABSTRACT}

A complete description is given of a set of all-glass rectifying stills, suitable for distillation at pressures ranging from atmospheric down to about $50 \mathrm{~mm}$. The stills are provided with efficient bubbling-cap columns containing 30 to 60 plates. Adiabatic conditions around the column are maintained by surrounding it with a jacket provided with a series of independent electrical heating units. Suitable means are provided for adjusting or maintaining the reflux ratio at the top of the column. For the purpose of conveniently obtaining an accurate value of the true boiling points of the distillates a continuous boiling-point apparatus is incorporated in the receiving system. An efficient still of the packed-column type for distillations under pressures less than $50 \mathrm{~mm}$ is also described.

Methods of operation and efficiency tests are given for the stills.

\section{CONTENTS}

I. Introduction.... 852

II. General features of the still assembly

III. Still pot_._. 853

IV. Filling tube and heater for the still pot.

V. Rectifying column

1. General features.... 856

2. Large-size column

3. Medium-size column...... 858

4. Small-size column

VI. Column jacket_...

VII. Reflux regulator

1. With variable reflux ratio..... 861

2. With constant reflux ratio.... 863

VIII. The continuous boiling-point apparatus

IX. Condensers and receiver... 867

X. The mounting of the still

XI. Manufacture and transportation of the bubbling-cap still _...... 870

XII. Operation of the bubbling-cap still

XIII. Columns for distillations at pressures less than $50 \mathrm{~mm}$

XIV. Stopcock lubricant

XV. Efficiency of the stills

XVI. Selection of a laboratory still

XVII. Acknowledgment .

Appendix 1. Regulation of the pressure within the still assembly

Appendix 2. A convenient mixture for testing the operating efficiency of a

$$
\text { distilling column }
$$

1 Presented before the joint session of the divisions of petroleum chemistry, gas and fuel chemistry, and industrial and engineering chemistry at the meeting of the American Chemical Society, Buffalo, N. Y., September 2, 1931.

This paper describes the distillation equipment developed in connection with The Separation, Identifi. cation, and Determination of the Chemical Constituents of Commercial Petroleum Fractions, listed as project No. 6 of the American Petroleum Institute Research. Financial assistance in this work has been received from a research fund of the American Petroleum Institute donated by John D. Rockefeller. This fund is being administerea by the institute with the cooperation of the central petroleum committee of the National Research Council.

3 Research associate for the American Petroleum Institute. 


\section{INTRODUCTION}

In order to separate a complex mixture, such as petroleum, as completely and as rapidly as possible by fractional distiflation, an efficient equipment is required. The principal requirements and characteristics of such a distillation unit are the following:

1. An efficient rectifying column. If the over-all vertical distance available is limited, for example, by the height of the laboratory room, the bubble-cap type of column should be employed.

2. Suitable means for adjusting and maintaining the reflux ratio at the top of the column.

3 . A device for determining accurately $\left( \pm 0.05^{\circ}\right.$ C. $)$ and continuously the true boiling point of the distillate as it is collected, in order that the "cuts" may be properly made.

4. Control of the nature of the atmosphere within the still, in order to prevent oxidation and minimize "cracking."

5. Exact control of the pressure under which the distillation takes place. This is desirable in order to eliminate the influence of variations in barometric pressure which will affect the boiling points of the distillates and thus require the application of corrections before cuts can be correctly made; and in order to permit distillations under reduced pressure when necessary.

6. Provision for exact temperature control of all parts of the system.

7. Provision for withdrawal of distillates without disturbing the operation of the still.

8. Appropriate protection against fire hazard when working with inflammable liquids.

The purpose of this paper is to describe in detail an all-glass still embodying the above characteristics, which has been developed during the past four years, and to give directions for the best operating procedure. While certain features of the still are new and constitute definite improvements over former practice, the general elements of design follow well-known principles. A battery of 10 stills of this type has been operated at the bureau on a continuous 24-hour schedule for the past 15 months with satisfactory results and without a single breakage or accident of any kind.

\section{GENERAL FEATURES OF THE STILL ASSEMBLY}

An assembly drawing of the still is shown in Figure 1. All parts are constructed of Pyrex laboratory glass. The still pot, 1 , is heated by means of an electric heater, 2 , which is connected in series with a rheostat. The still pot and its heater are thoroughly insulated from the surroundings by means of a brass container, 3 , the inside and top of which are covered with asbestos sheet. This method of heating the still pot has the advantage that in case of an accidental breakage during distillation of inflammable liquids, a fire would be confined principally to the metal container.

The pot is charged through the filling tube, 4. A thermometer is suspended in the still pot through the arm, 5 , which is provided with a special mercury seal. The closed side arm on the right is provided for the purpose of attaching a gas inlet, if distillations are to be carried out in an atmosphere of inert gas. 


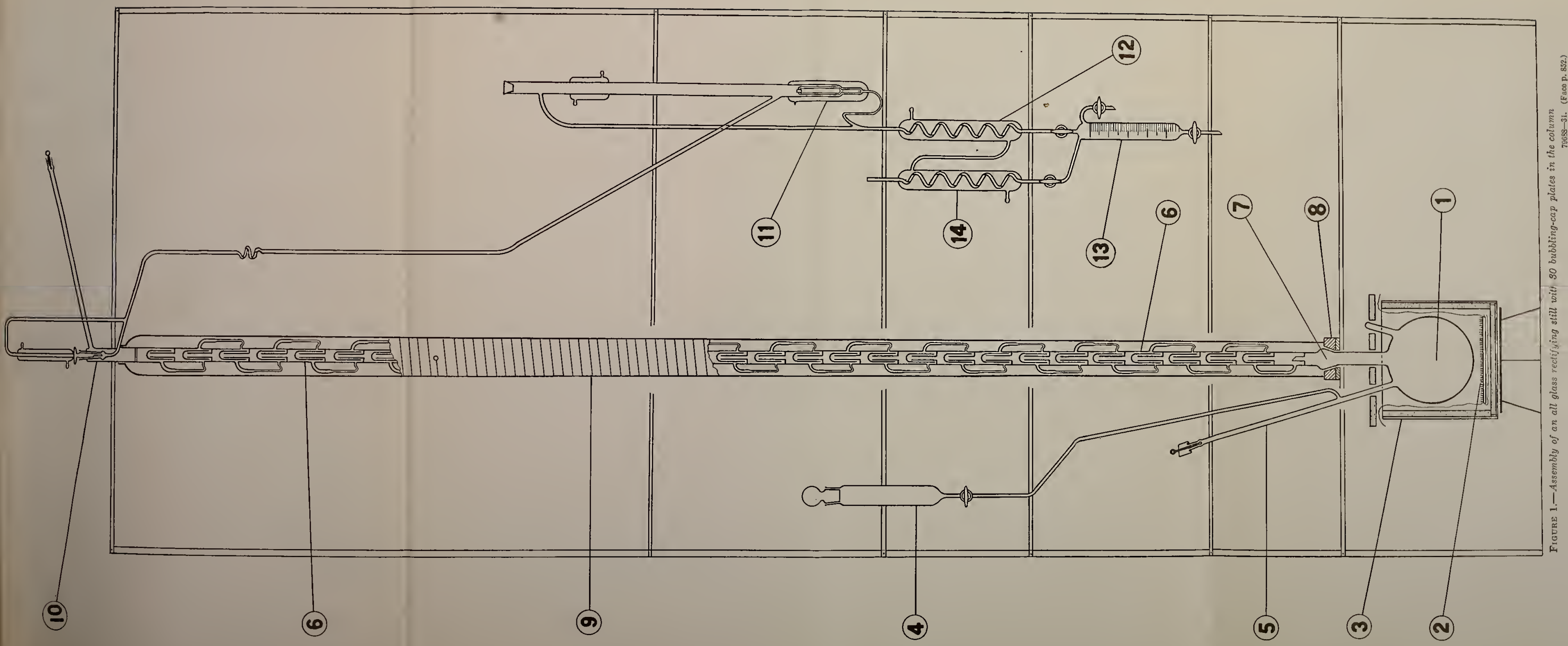


The rectifying column, 6 , consists of 30 to 60 bubbling-cap plates.

The column is sealed to the still pot by means of a connecting joint, 7 , which is firmly supported at 8 .

The column jacket, 9 , is also carried on support 8 . Around this jacket several electric heating coils are wound, each of which is independently controlled by means of a rheostat. During the distillation, a slow current of air passes through the column jacket, entering at the top and leaving at the bottom. Through a vertical opening at the top of the jacket (fig. 8), thermometers are suspended in the centers of the respective heated sections. With this arrangement it is possible to maintain a desired temperature gradient along the column.

The reflux head, 10, is sealed to the top of the column. Two types of reflux heads are in use, the one shown in Figure 1 is for a variable reflux ratio (for details see fig. 9); the other (for details see fig. 10) is for a constant reflux ratio. The reflux head is provided with a tube for a thermometer, so that the temperature at the top of the column can be determined during the distillation. For accurate work, however, the reading of the "condensation temperature" has been abandoned. Instead a continuous boiling-point apparatus, 11, is used for determining the true boiling points of the fractions as they are collected.

After leaving the boiling-point apparatus, the liquid condensate enters the cooler, 12 , where it is cooled and delivered to the receiver, 13 , which is provided with a return condenser, 14, the outlet of which is connected to the pressure regulator. (See Appendix I.) Stopcocks are located between the condenser and the receiver, so that during a vacuum distillation fractions can be withdrawn from the receiver without breaking the vacuum in the still proper.

\section{STILL POT}

The still pot shown in Figure 2 consists of a round-bottomed flask, the walls of which are extra heavy and of uniform thickness. Its size will, of course, depend upon the quantities of material to be distilled. The pot is provided with two side arms, one for the thermometer and filling tube and one for the gas inlet, which is used if the distillation is carried out in an atmosphere of inert gas.

The thermometer tube is provided with a ground joint and a mercury seal, which are shown separately in Figure 2. The bottom of the mercury cup is depressed in order to prevent any mercury from entering the still pot when the thermometer is removed. For this purpose most of the mercury in the cup is first sucked out after which the remaining traces are brushed down into the bottom of the cup. The gas inlet shown in Figure 2 is made of three coaxial tubes. This is stronger than the usual ring seal and will prevent breakage if bumping occurs in the still pot.

\section{FILLING TUBE AND HEATER FOR STILL POT}

The filling tube is shown in Figure 3 . The $4 \mathrm{~mm}$ stopcock at the bottom of the filling tube should have a Pyrex plug and be carefully ground in order to prevent leakage during the distillation.

The heater is made of an octagonal piece of hard asbestos board ( $3 \mathrm{~mm}$ thick) provided with saw cuts for holding the spiral heating 


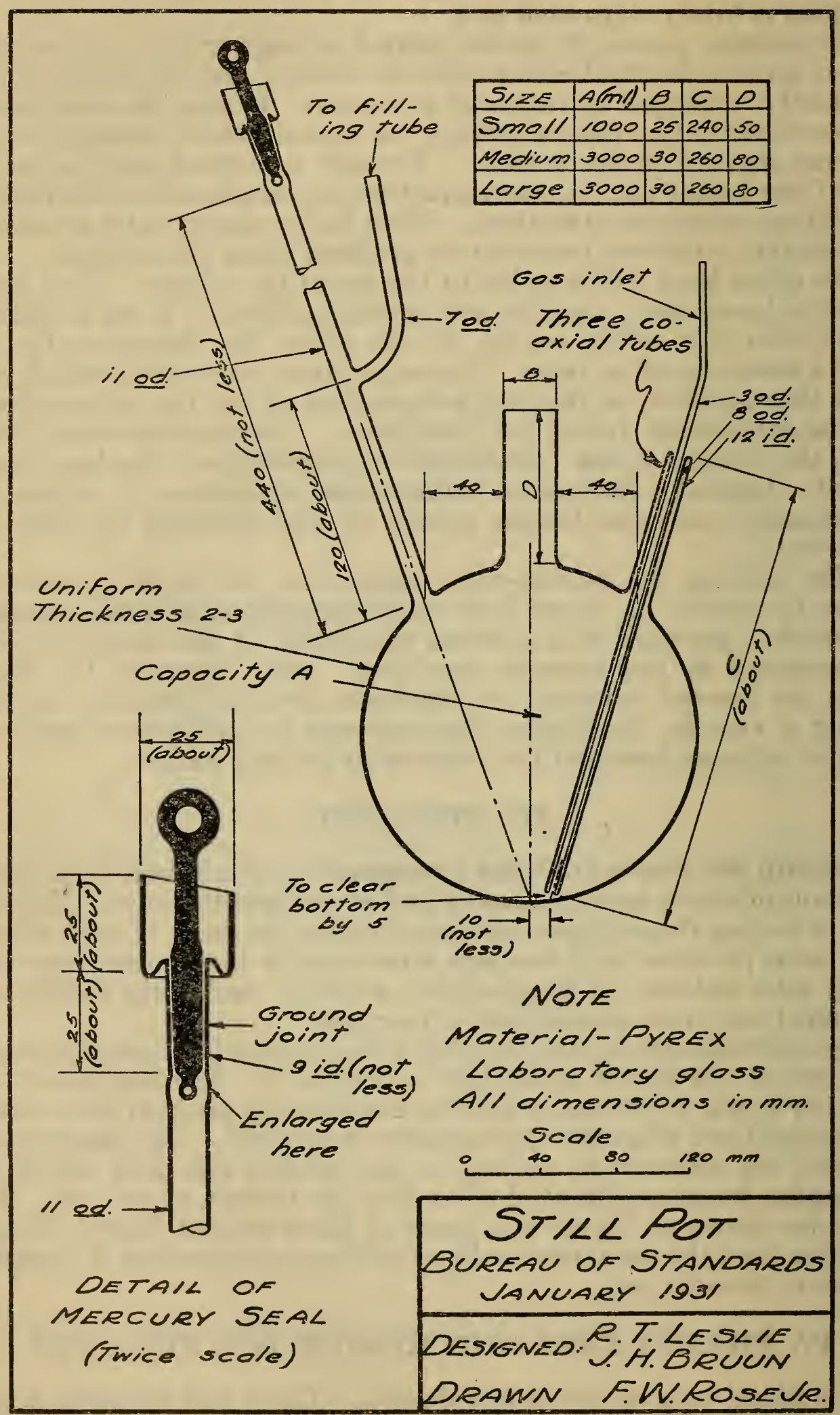

Figure 2.-Still pot 


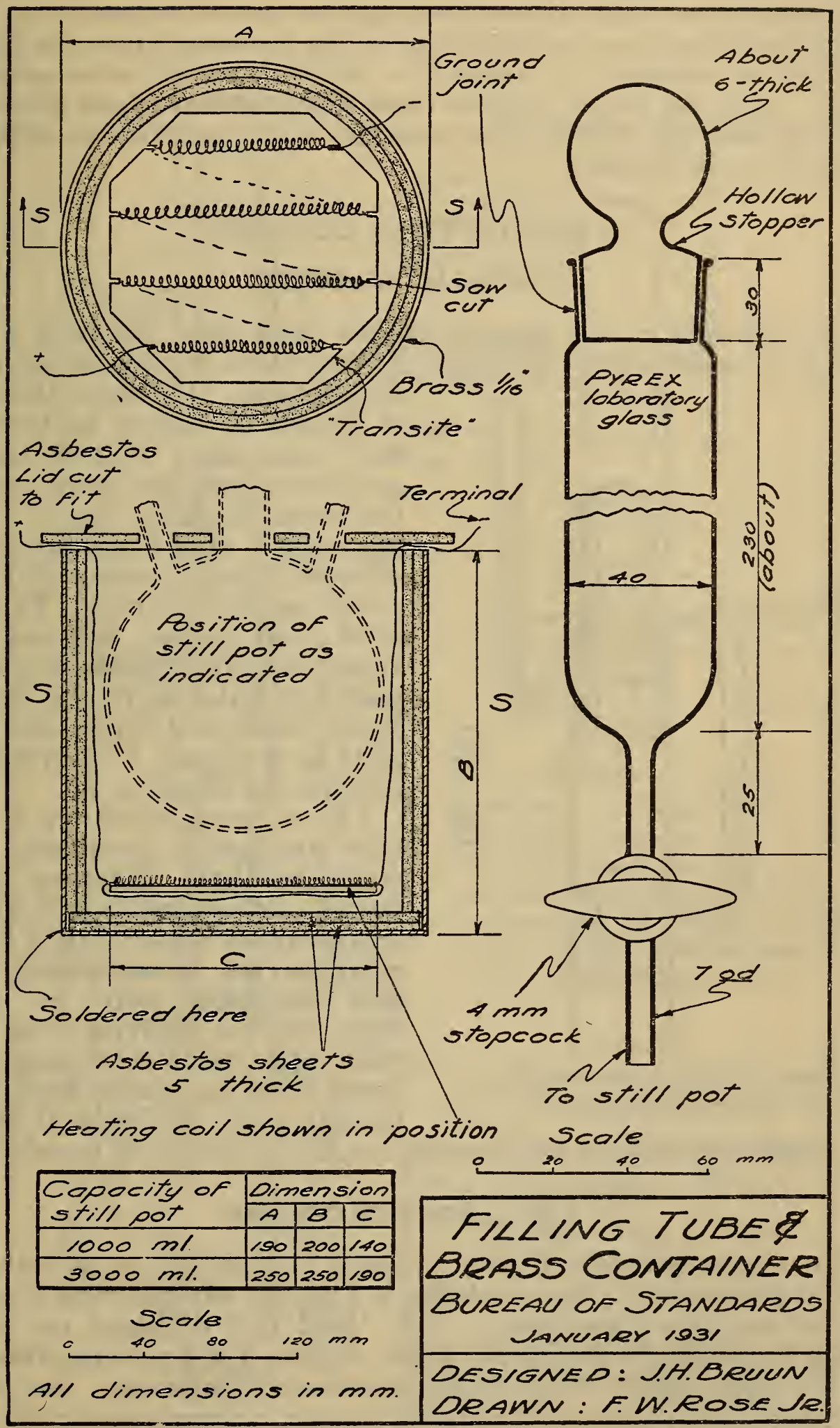

FIgURE 3.-Filling tube and brass container 
coils. (See fig. 3.) If about 7 of Chromel A heating wire No. 22 is used with a 3 -liter pot and a 110 -volt circuit, sufficient heat for the distillation is provided without heating the wire to redness.

The heater is placed on the bottom of the brass container, the inside of which is insulated by means of a double layer of $5-\mathrm{mm}$ asbestos sheet. After the heater and the brass container have been placed in position under the still pot, the container is covered with an asbestos lid as shown in Figure 3.

\section{RECTIFYING COLUMN}

\section{GENERAL FEATURES}

The principle of the column will be clear from Figure 4 . The ascending vapor enters the column at the bottom, where a liquid seal,

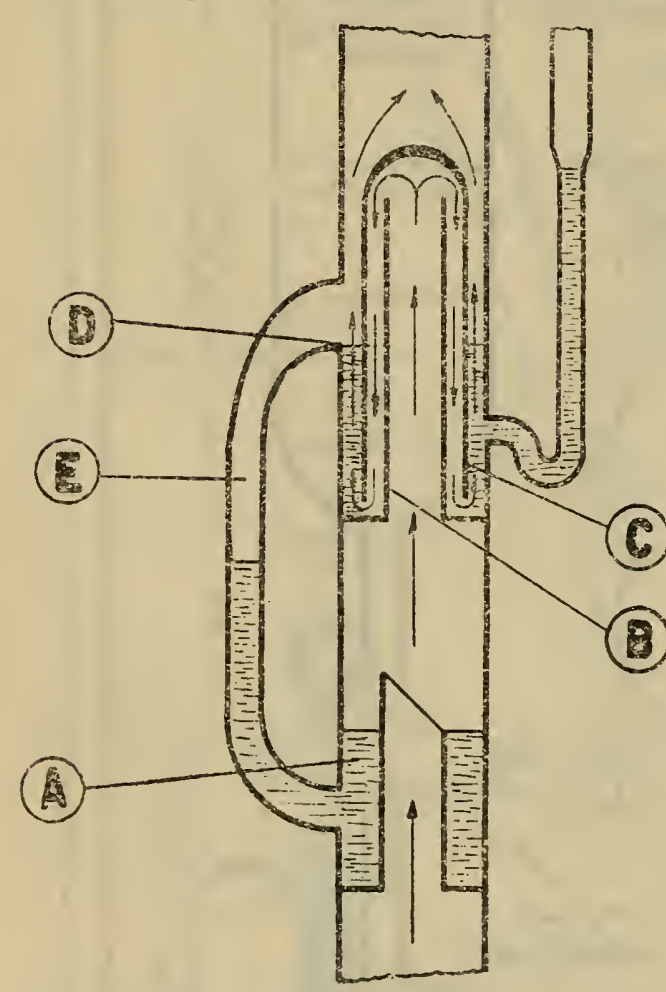

Figure 4.-Explanatory diagram of the rectifying column

$A$, is located. It follows the path of the arrows passing up through the "vapor riser," $B$, in the center and down along the inside wall of the loose bubbling cap, $C$. At the bottom of the cap the vapor is forced through narrow slits in a stream of fine bubbles. This insures good contact between the vapor and liquid phases as well as thorough agitation of the liquid. When condensed liquid fills the plate to the point $D$ it overflows through the side arm $E$.

The columns, which are of the type previously described, ${ }^{4}$ are made in three different sizes (large, medium, and small), which are described below. All of these columns are nonsiphoning ${ }^{4}$ so that the stills may be shut down for the night or over the week end without drainage of liquid from the plates back into the still pot. Similarly, if flooding should necessitate an interruption, the column will remain full.

\section{THE LARGE-SIZE COLUMN}

The column shown in Figure 5 is used for distillations at rates up to $5 \mathrm{ml}$ per minute ${ }^{5}$ (the usual rate is $2 \mathrm{ml}$ per minute) with a reflux ratio of not less than $10: 1$. The "hold up" of liquid per plate during the distillation is about $5 \mathrm{ml}$. Figure 5 is a working drawing for the glass blower with all the necessary dimensions, the most

4 J. H. Bruun. Ind. Eng. Chem. Anal. ed., vol. 1, p. 212; 1929.

$\checkmark$ This, of course, is equivalent to more than $50 \mathrm{ml}$ of liquid per minute returning through the column. 


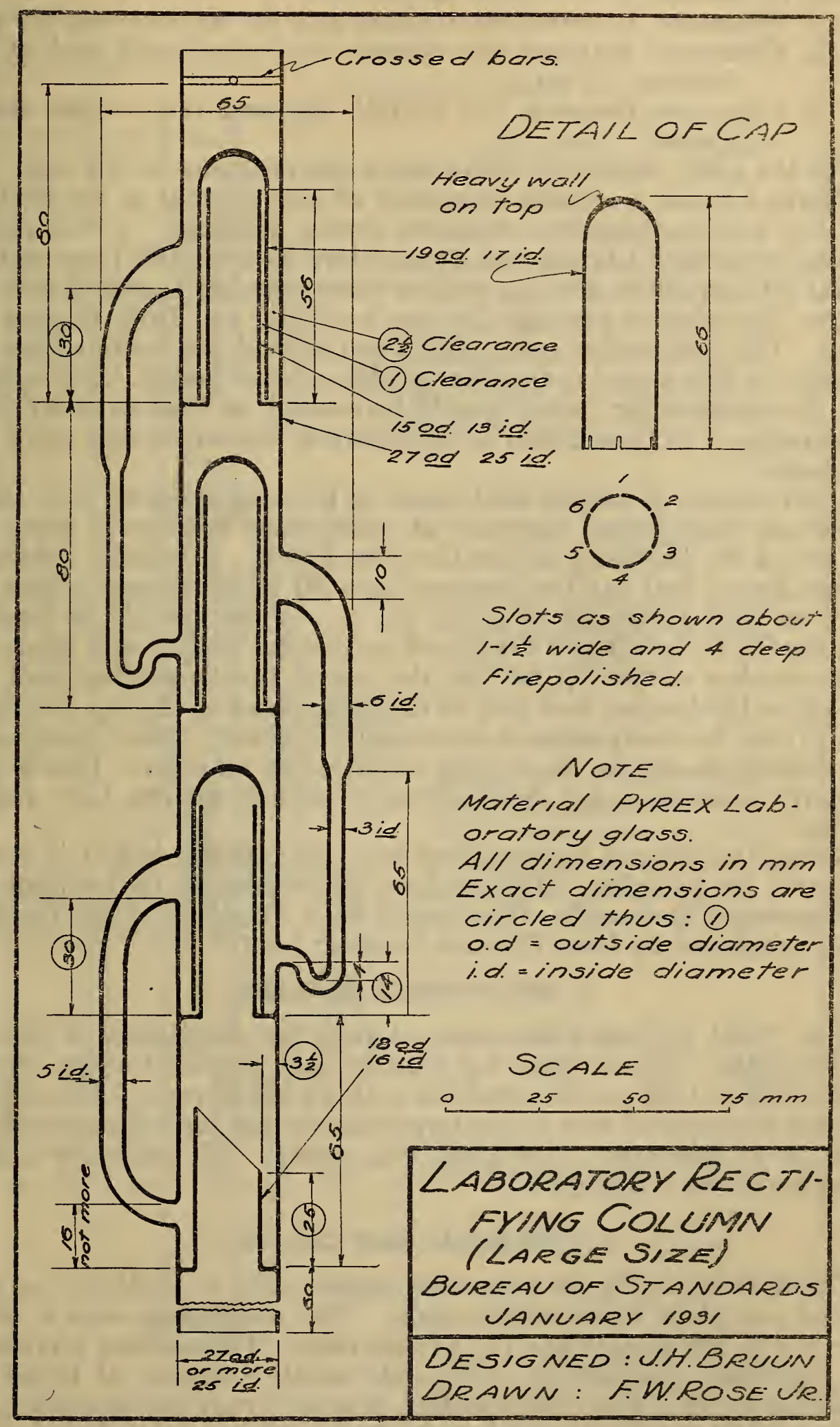

FIGURE 5.-Large-size column 
important ones being inclosed in circles. The following clearances should be adhered to as closely as possible:

1. Clearance between the column and the bubble cap, $2.5 \mathrm{~mm}$.

2. Clearance between the column and the liquid seal at the bottom, $3.5 \mathrm{~mm}$.

3. Clearance between the bubble cap and the "vapor riser," $1 \mathrm{~mm}$.

For the other important dimensions the tolerance is $\pm 1 \mathrm{~mm}$.

Figure 5 shows a column consisting of a liquid seal at the bottom, one top section, and two standard center sections. A "30-plate" column consists of 1 liquid seal, 29 standard sections and 1 top section. About $80 \mathrm{~mm}$ above the top plate a glass crossbar is sealed into the column in order to prevent the top bubbling cap from leaving the plate. The return-flow arms should not extend too far out from the column, as this would necessitate a wide column jacket. The column and the surrounding jacket should be coaxial, so that at every place a clearance of at least $2 \mathrm{~mm}$ is left between the return-flow arms and the jacket.

A tall column is usually first made up in sections (of $80 \mathrm{~mm}$ each). These are then joined together at some place between a plate and the top of the bubbling cap on the plate below. A column consisting of one liquid seal (at the bottom) and 30 bubbling-cap plates will have an over-all height of about $2.4 \mathrm{~m}$. A distance of at least 60 $\mathrm{mm}$ should be left below the liquid seal at the bottom and above the glass crossbar at the top, so that the seal to the connecting joint (see fig. 15) at the bottom and that to the reflux head at the top (see figs. 9 and 10) can be made without reheating the plates. After completion, the column should be thoroughly annealed in an oven. This is particularly important for distillations of liquids having high boiling points.

Before the column is assembled into the column jacket it should be tested for pinholès. This is done by evacuating the column and testing with a Tesla coil. (For details see S. Dushman, High Vacuum, p. 81, published by Gen. Electric Review; 1922.)

\section{MEDIUM-SIZE COLUMN}

The "hold up" of this column during the distillation is about 4 $\mathrm{ml}$ per plate. This column has usually been operated at an average rate of about $1 \mathrm{ml}$ per minute with a $10: 1$ reflux ratio. The column is made in the same way as the large-size column, and the same directions as to tolerances, annealing, etc., apply. All necessary dimensions are given in Figure 6.

\section{THE SMALL-SIZE COLUMN}

The drawing in Figure 7 shows a column with a "hold up" of only $0.6 \mathrm{ml}$ per plate when in operation. The distillation rate is about $0.1-0.2 \mathrm{ml}$ per minute at a $10: 1$ reflux ratio. This column is intended for fractional distillations when only small amounts of liquid are available or for a final fractionation of nearly pure compounds. One plate section of this column is only $40 \mathrm{~mm}$ high, so that in a laboratory of average height (about $3.5 \mathrm{~m}$ ) it is possible to mount a rectifying column containing about 60 plates. All necessary dimensions for the small column are given on the drawing. The general specifications given for the large column also apply to this one. 


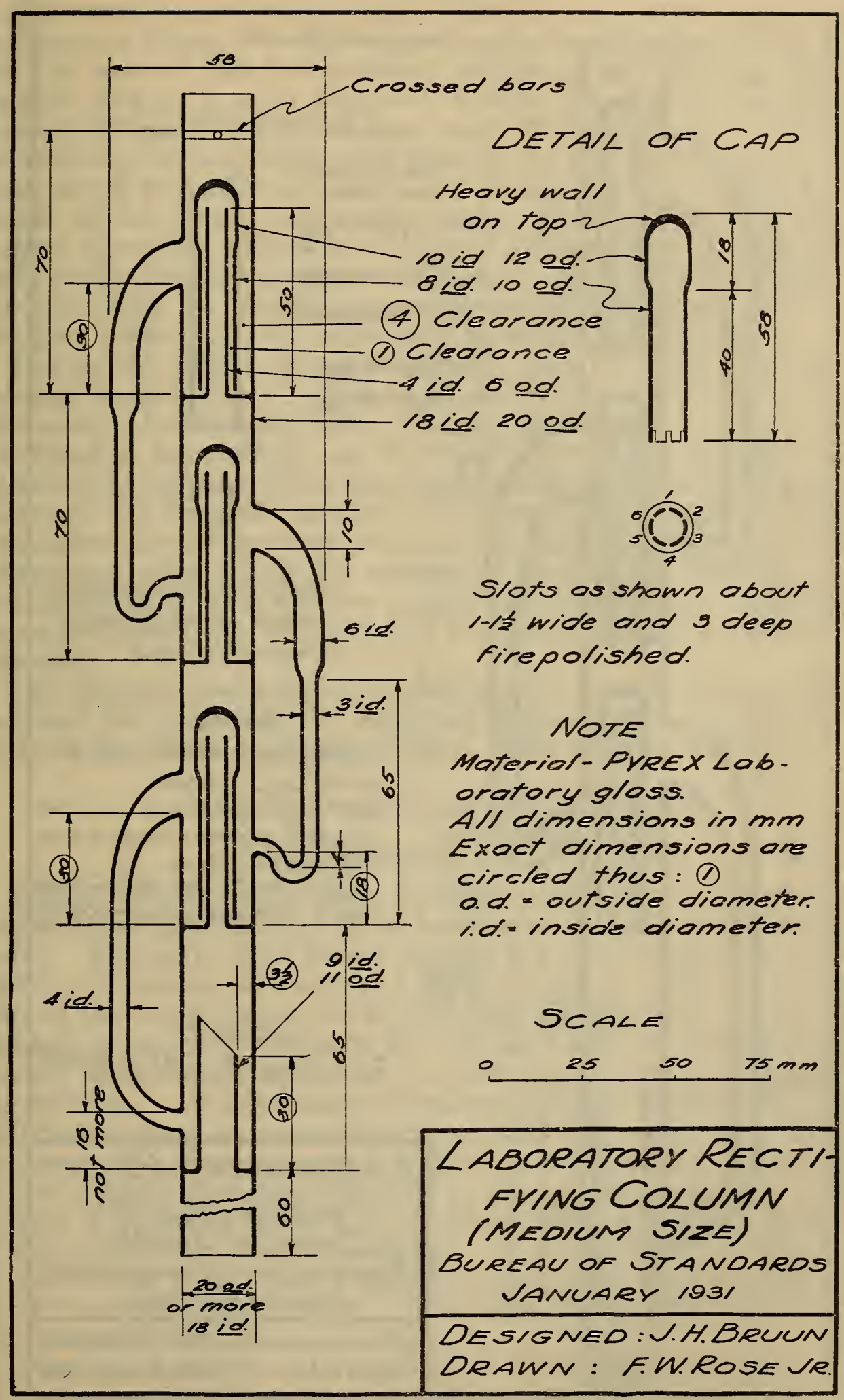

Figure 6.-Medium-size column

$79688-31-7$ 


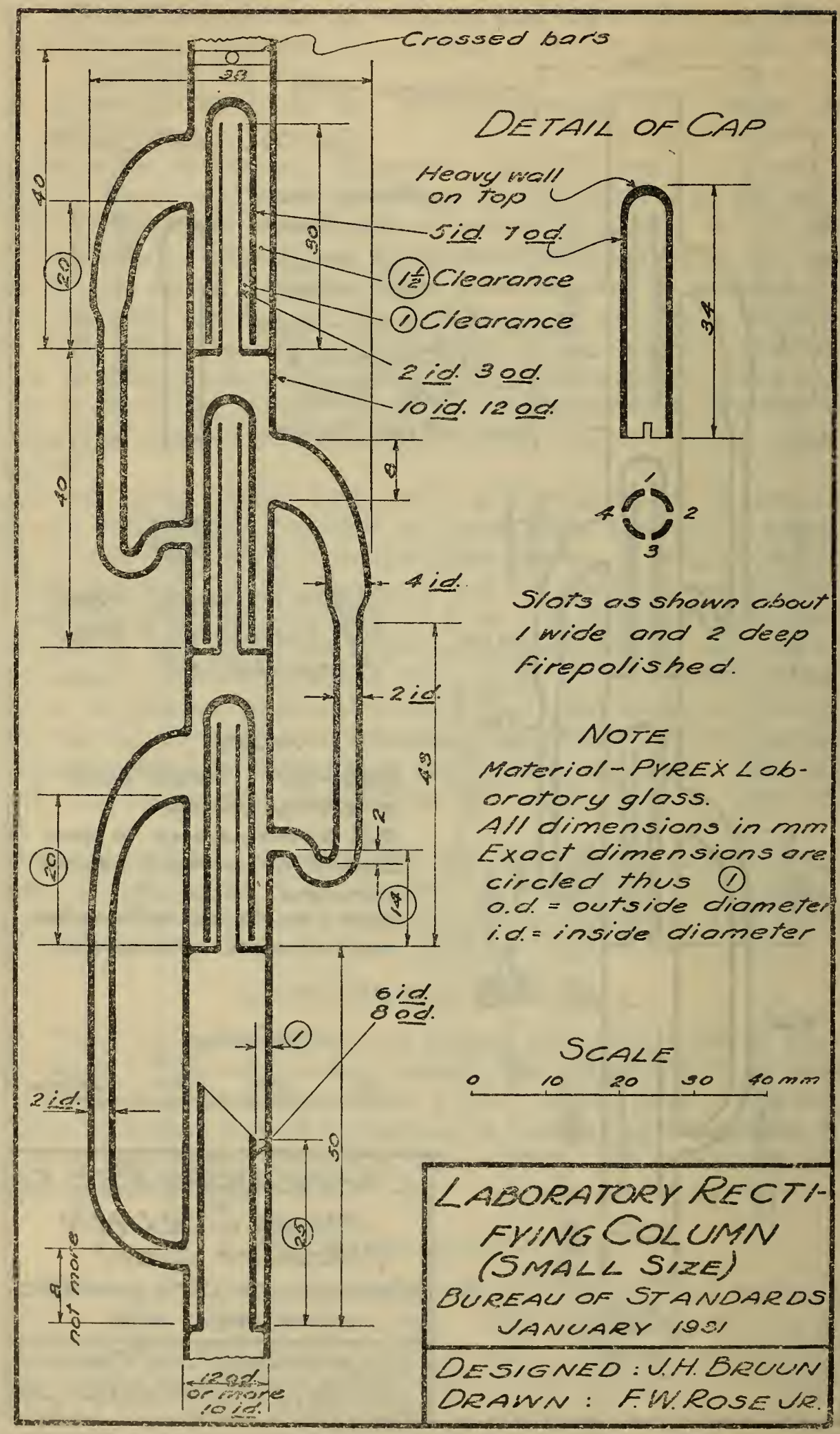

FIgURE 7.-Small-size column 


\section{THE COLUMN JACKET}

The rectifying column is surrounded by the column jacket, which is shown in Figure 8. Eight glass knobs, $K$, are attached for fastening the ends of four independent heating units. Each heating unit consists of 6 to $7 \mathrm{~m}$ of Chromel A wire No. 22 wound uniformly around the jacket from one glass knob to the next one. In order to prevent the heating coils from slipping on the smooth glass surface, two pieces of asbestos cord (6 mm thick) are laid along the entire length of the jacket on opposite sides. The heating wires are then wound over this cord before the still is assembled. For operation each heating section is connected in series with an independent rheostat (50 ohms for distillations at temperatures below $100^{\circ} \mathrm{C}$, and $16 \mathrm{ohms}$ above $100^{\circ} \mathrm{C}$., when used on a 110 -volt circuit).

During the distillation a slow stream of air is forced into the jacket through the side arm, $I$. The air leaves the jacket through the bottom side arm, $O$. The inlet tube, $I$, as well as the outlet tube, $O$, should be attached tangentially, as shown in Figure 8, so that the cold air entering the jacket will not impringe directly on the rectifying column. Through the vertical opening, $T$, at the top of the column jacket, four thermometers or thermocouples (one for each heating section) are suspended, after which the opening is closed with asbestos. After the rectifying column and the column jacket have been assembled the space between them at the top of the jacket should be packed with asbestos.

The diameter and height of the column jacket will depend upon the size and the height of the rectifying column; these dimensions are given in the Table of Figure 8 for the three sizes of column.

\section{THE REFLUX REGULATOR}

\section{WITH VARIABLE REFLUX RATIO}

Figure 9 shows a still head ${ }^{6}$ for variable reflux. In this device the vapor enters from the top of the column at $A$ and is divided into two parts. The major part passes up into the condenser, $B$, and is completely condensed and returned to the column, while the remaining. part of the vapor leaves the column through side arm, $T$, and passes through stopcock $S$ and tube $L$. The side arm, $T$, should be sloped toward the column, so that any liquid condensed in it will return to the column. An advantageous feature of this regulator is that the reflux ratio may be increased or decreased by turning the lubricated stopcock, $S$. (For stopcock lubricant see Sec. XIV.) In the beginning of a distillation this stopcock is kept closed and the rectifying: column is operated under a total reflux until equilibrium conditions in the column have been established. The stopcock is then adjusted to the desired point, after which it is sealed externally with collodion as a precaution against leaks. This regulator also works satisfactorily for immiscible liquids.

If desired a thermometer may be placed at the top of the column through the tube $W$, which is inclined about $60^{\circ}$ from the vertical in order to allow more height for the column. This tube is provided with a ground joint at the top.

6 A reflux head of a somewhat similar design has been described by IV. J. Marshall, Ind. Eng. Chem., vol. 20, p. 1379; 1928 . 


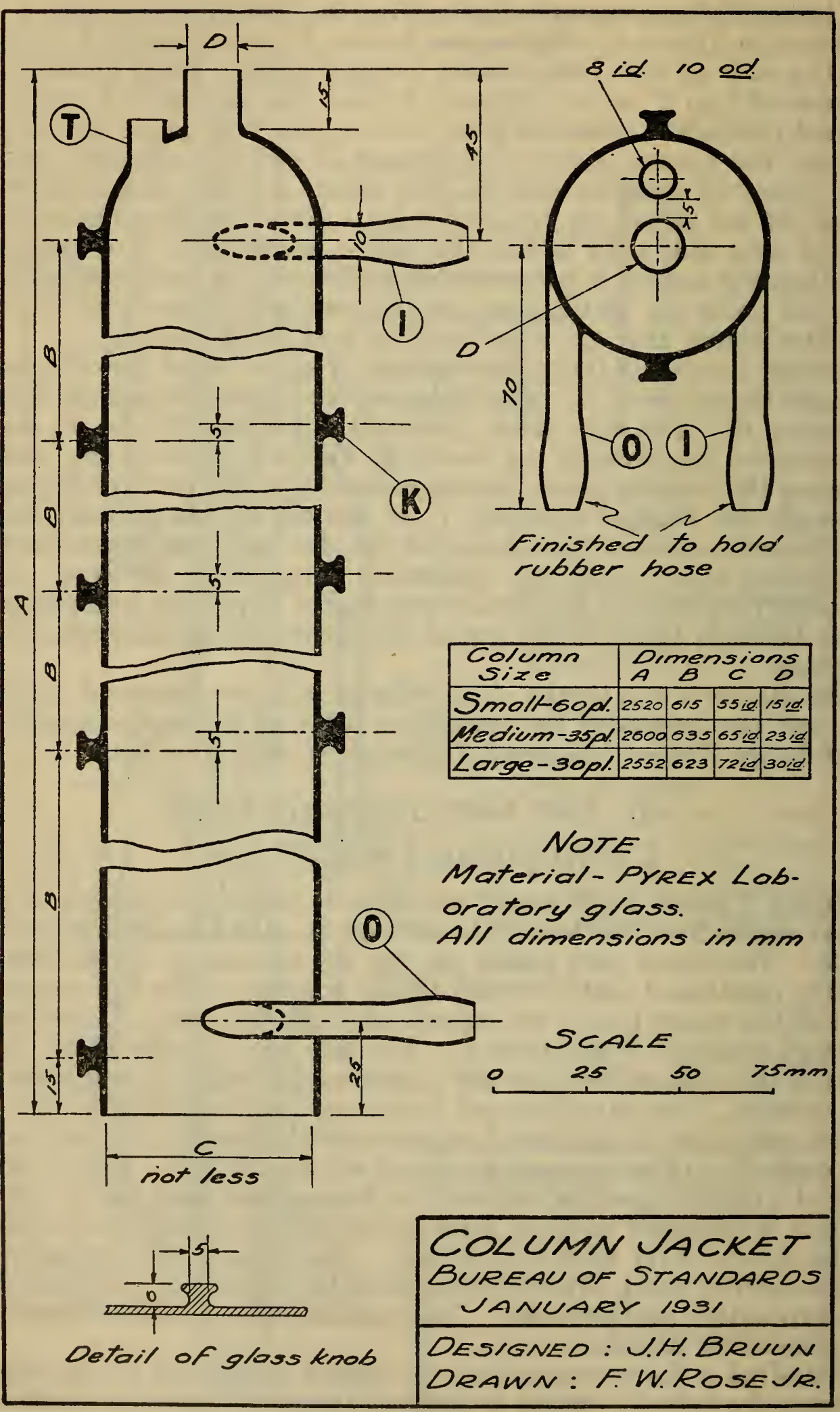

FIGURE 8.-Column jacket 


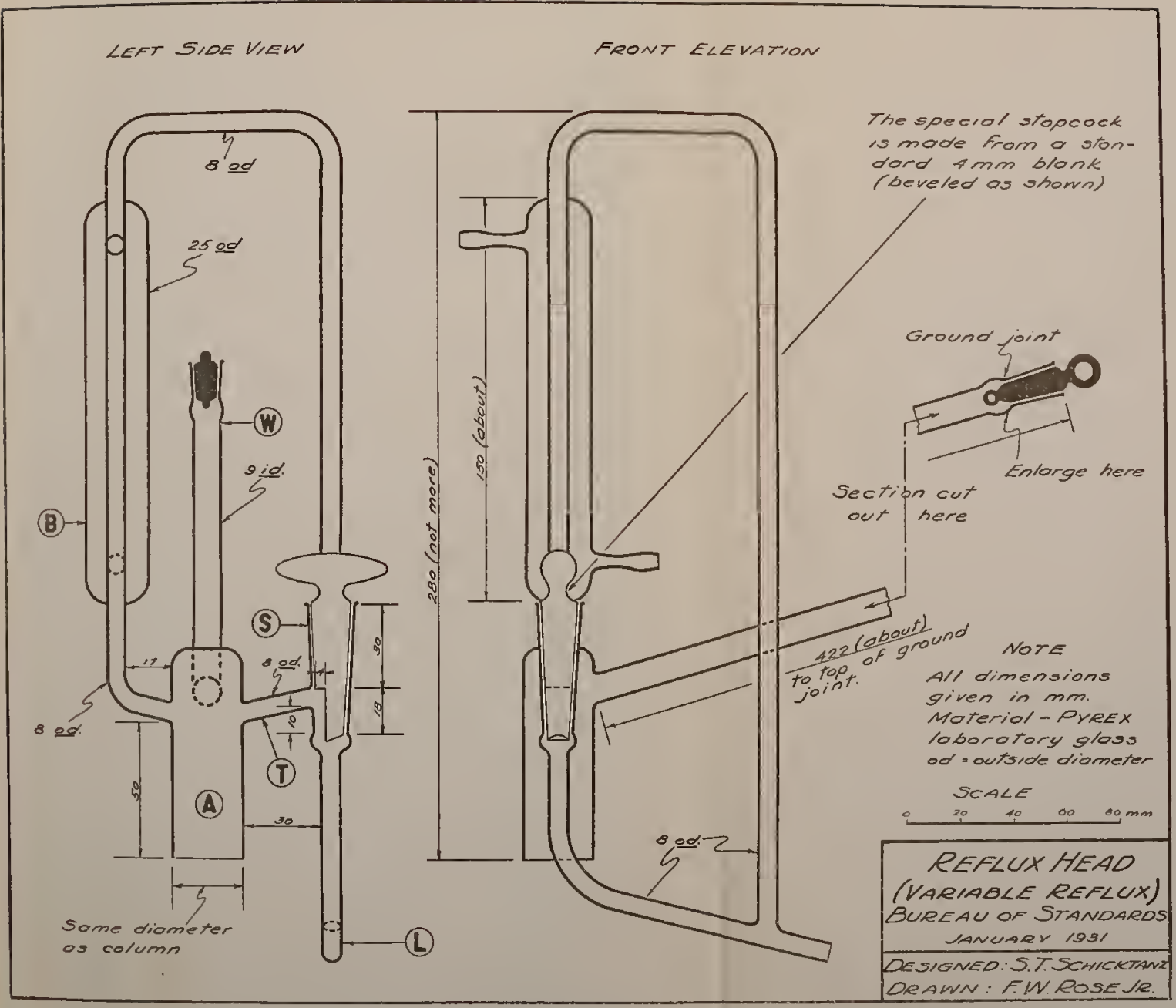

FIGURE 9.-Reflux regulator for variable reflux ratio

70658-31. (Face p. 862.) No. 1 


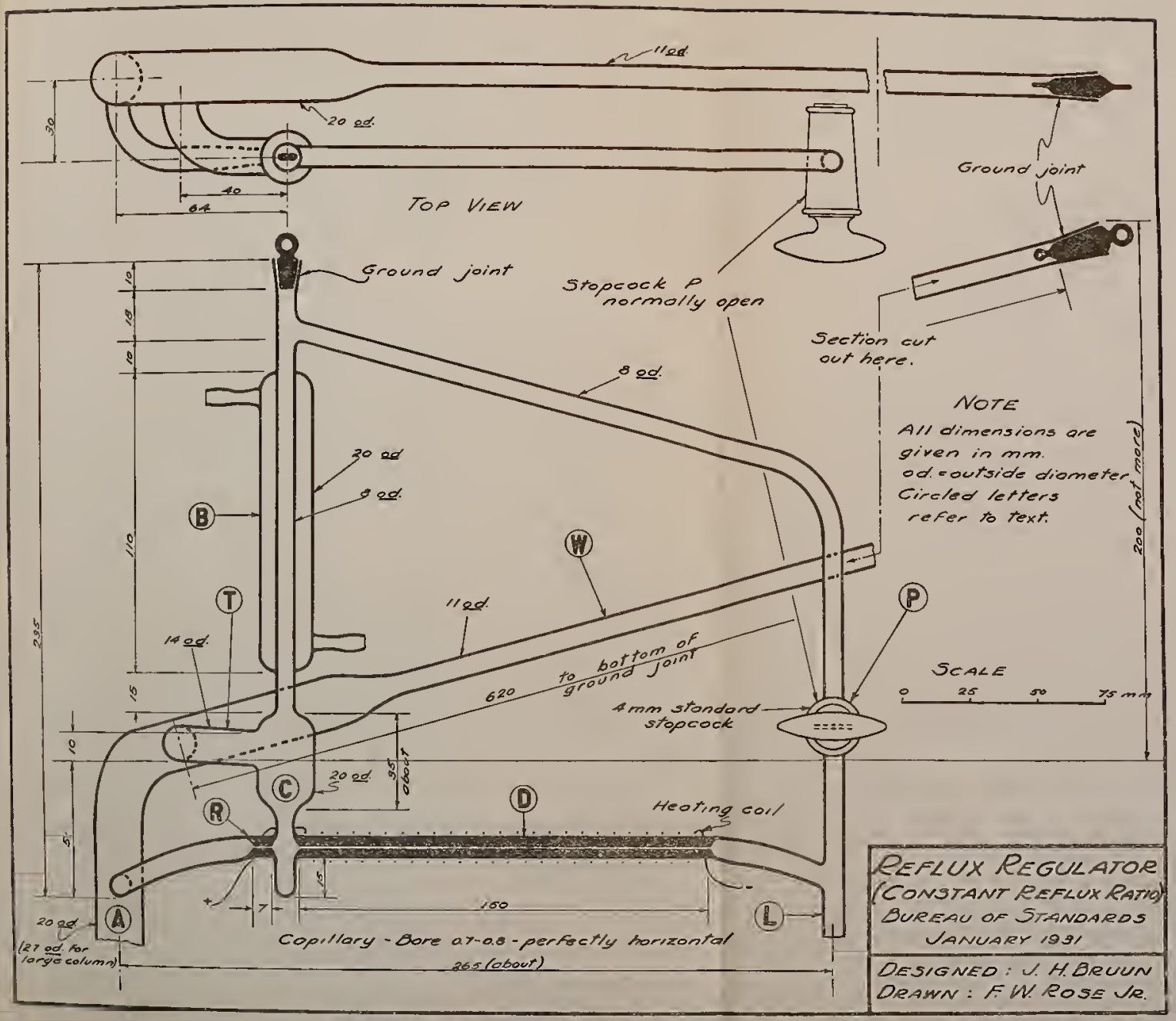

FIOURE 10.-Reflux regulator for constont $(10: 1)$ reflux ratio

i8688-31, (Face pı 862.) No. 2 


\section{WITH CONSTANT REFLUX RATIO}

Figure 10 shows a working drawing of a reflux regulator previously described. $^{7} \quad$ It is intended for distillations during which a constant and definitely known reflux ratio (mass of vapor returned as refluxed liquid to the column, divided by mass of distillate) is desired. The vapor from the rectifying column enters the reflux regulator at $A$ and passes through the side tube, $T$. By means of the condenser, $B$, it is then completely condensed into the bulb, $C$, from the bottom of which a certain part (90 per cent.) of the condensate is returned as refluxed liquid to the column through the shorter capillary tube, $R$. Another part (10 per cent) passes out as distillate through the longer capillary tube, $D$, and the tube, $L$.

Such a regulator with the dimensions shown in Figure 10 maintains a constant reflux ratio of 10:1 for any rate of distillation. If desired, however, flexibility in the reflux ratio can be obtained by placing a heating coil around the capillary tube, $D$, thus increasing the flow through this tube by decreasing the viscosity of the liquid.

An advantageous feature of this regulator is the fact that it offers no contact between greased stopcocks and hot vapor or liquid. Thus a contamination of the distillate with stopcock lubricant is prevented, and for vacuum distillations leaks at this point are avoided. On the other hand, this regulator is not suitable for distillation of immiscible liquids (such as a mixture of hydrocarbon with water) because of clogging of the capillary.

The thermometer tube, $W$, may be omitted if desired. This simplifies the construction because $T$ and $R$ in this case may be straight tubes instead of curved. A sketch of this modified form is shown in Figure 11.

\section{THE CONTINUOUS BOILING-POINT APPARATUS}

For the purpose of conveniently obtaining an accurate value of the true boiling point of the distillate instead of the "condensation point" which is the usual one obtained at the top of a rectifying column, a continuous boiling-point apparatus is incorporated in the assembly. This apparatus is of the Cottrell ${ }^{8}$ type and has been described in a previous paper. ${ }^{9}$ An assembly drawing of this apparatus is shown in Figure 12. The liquid from the reflux regulator at the top of the distilling column enters the apparatus at $A$. The top of the apparatus is closed by means of a stopper, which is sealed externally with a high-melting wax. Through this stopper two heavy copper or silver wires lead down to the heating coil, $H$ (Chromel A or platinum wire), by means of which the liquid in the bell, $B$, is kept boiling continuously. A mixture of vapor and liquid ascends from the bell, $B$, through two "pumping tubes," $N$, and is discharged against the thermometer, $T$, which is suspended from the stopper through the condenser $C$. The liquid leaves the apparatus at the bottom and overflows continuously through $O$ in to the cooler and receiver. (See assembly drawing in fig. 1.) The top of the apparatus is connected directly with $O$, by means of an equalizing line $E$.

7 J. H. Bruun, Ind. Eng. Chem., Anal. Ed., vol. 2, p. 187; 1930.

F. G. Cottrell, J. Am. Chem. Soc., vol. 41, p. $721 ; 1919$.

J. H. Bruun and M. M. Hicks-Bruun, B. S. Jour. Research, vol. 6, p. 871; 1931. 
A $4 \mathrm{~mm}$ stopcock may be sealed into the equalizing line, $E$, in order to facilitate the removal of the liquid from the apparatus by suction applied at $O$. The lower part of the apparatus is surrounded by a vacuum jacket, $V$, which should extend well above the top of the pumping tubes, $N$.

A working drawing of the apparatus with all the necessary dimensions is shown in Figure 13. It should be noted that the height of the pumping tubes ( $N$ in fig. 12) is given as $110 \mathrm{~mm}$ in Figure 13. This height has been found to be sufficient for the lower boiling

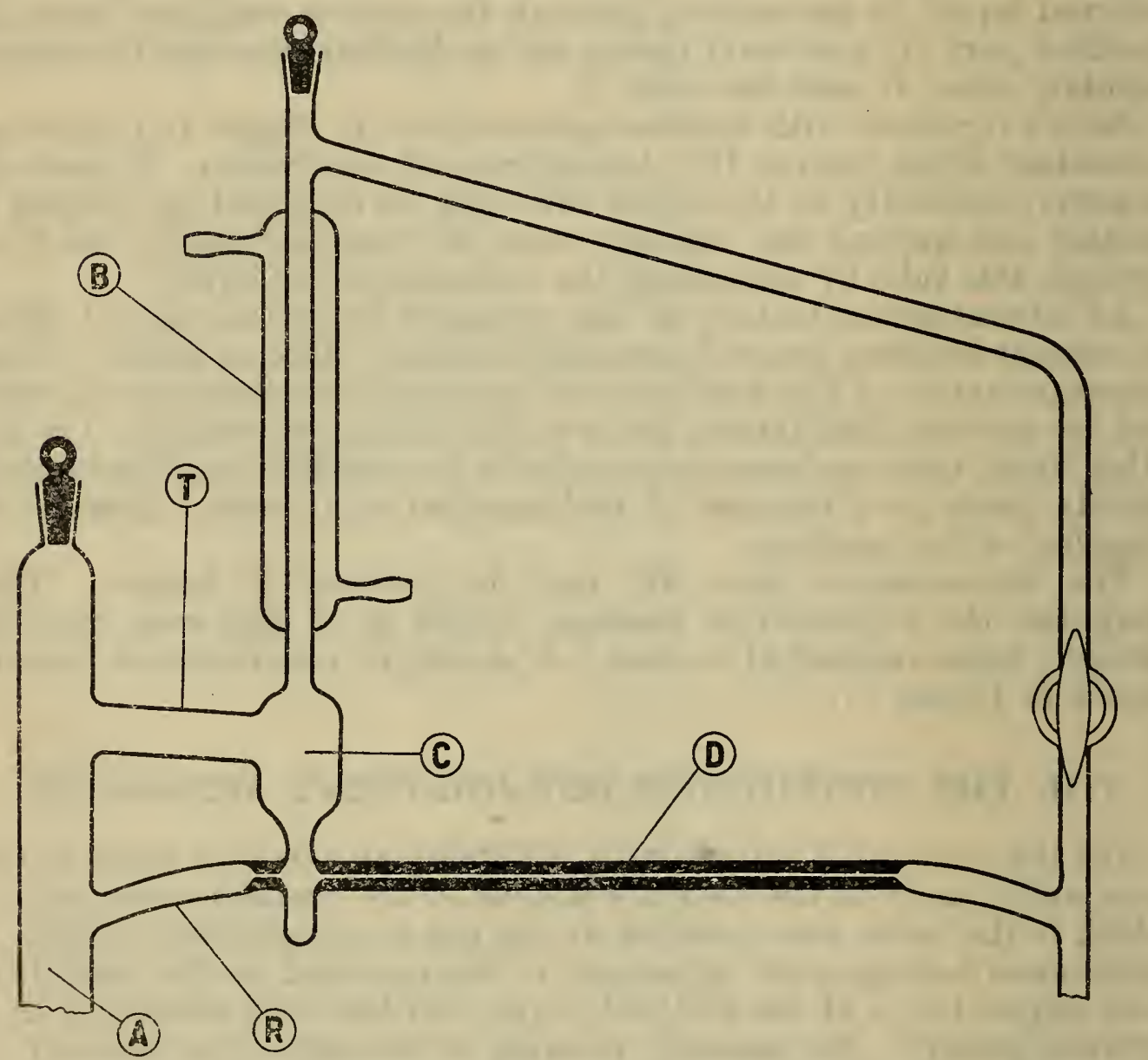

FigdRE 11.-Reflux regulator for constant reflux ratio. (Without the thermometer tube)

petroleum fractions, but should probably be increased for more viscous liquids, in order to dissipate superheat. ${ }^{10}$

A more elaborate construction of this apparatus is to replace the stopper at the top of the apparatus with a ground-glass joint provided with two tungsten leads. An improved modification of this type has been used on one rectifying still. For most purposes, however, it was not considered necessary to go to the extra expense of using the ground-joint top. For this reason the simplified form of construction is shown in the figure.

10 On this point see further, Washburn and Read, J. Am. Chem. Soc. vol. 41, p. 731, par. 1; 1919. 


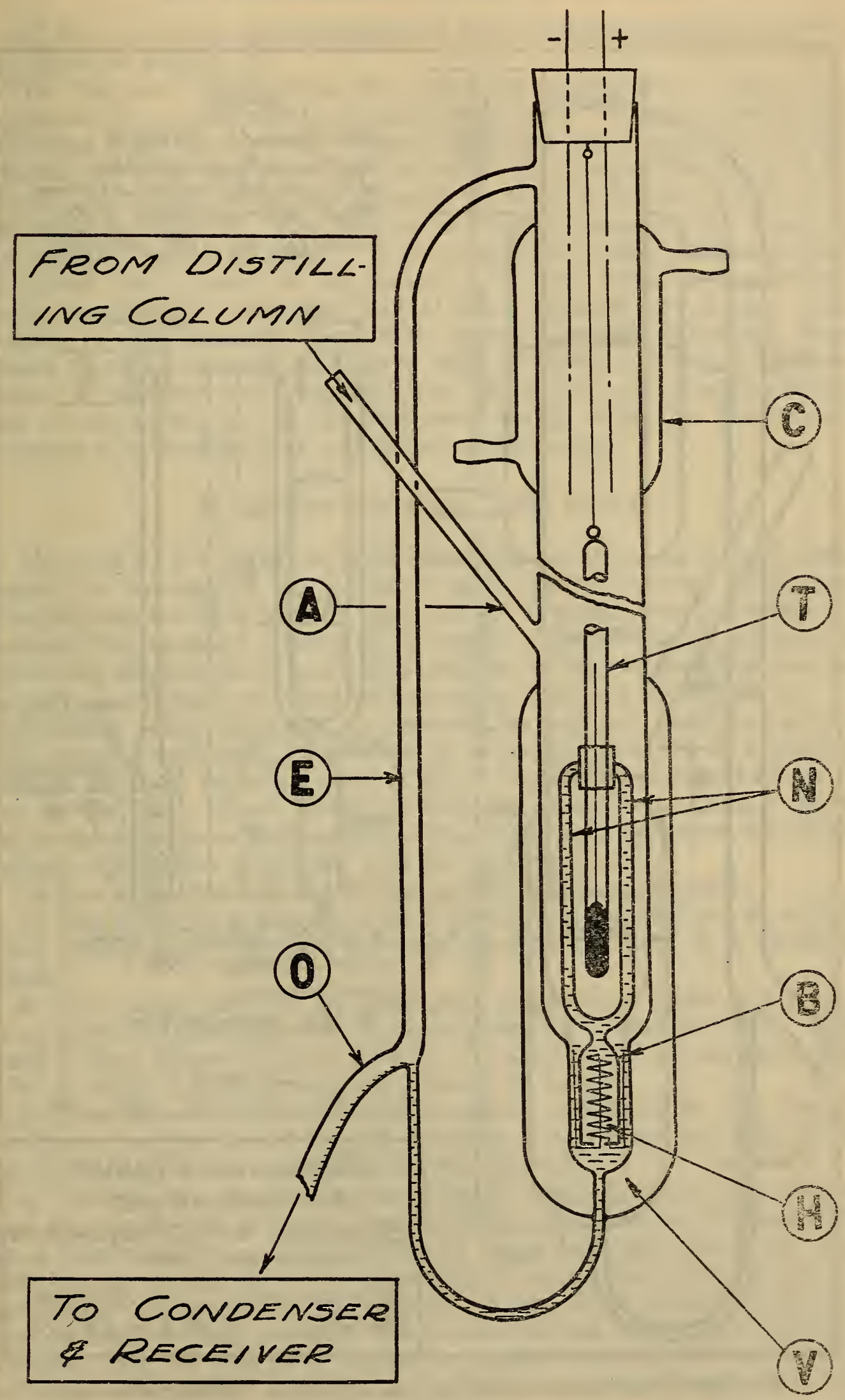

FIGURE 12.-Continuous boiling-point apparatus (explanatory diagram) 


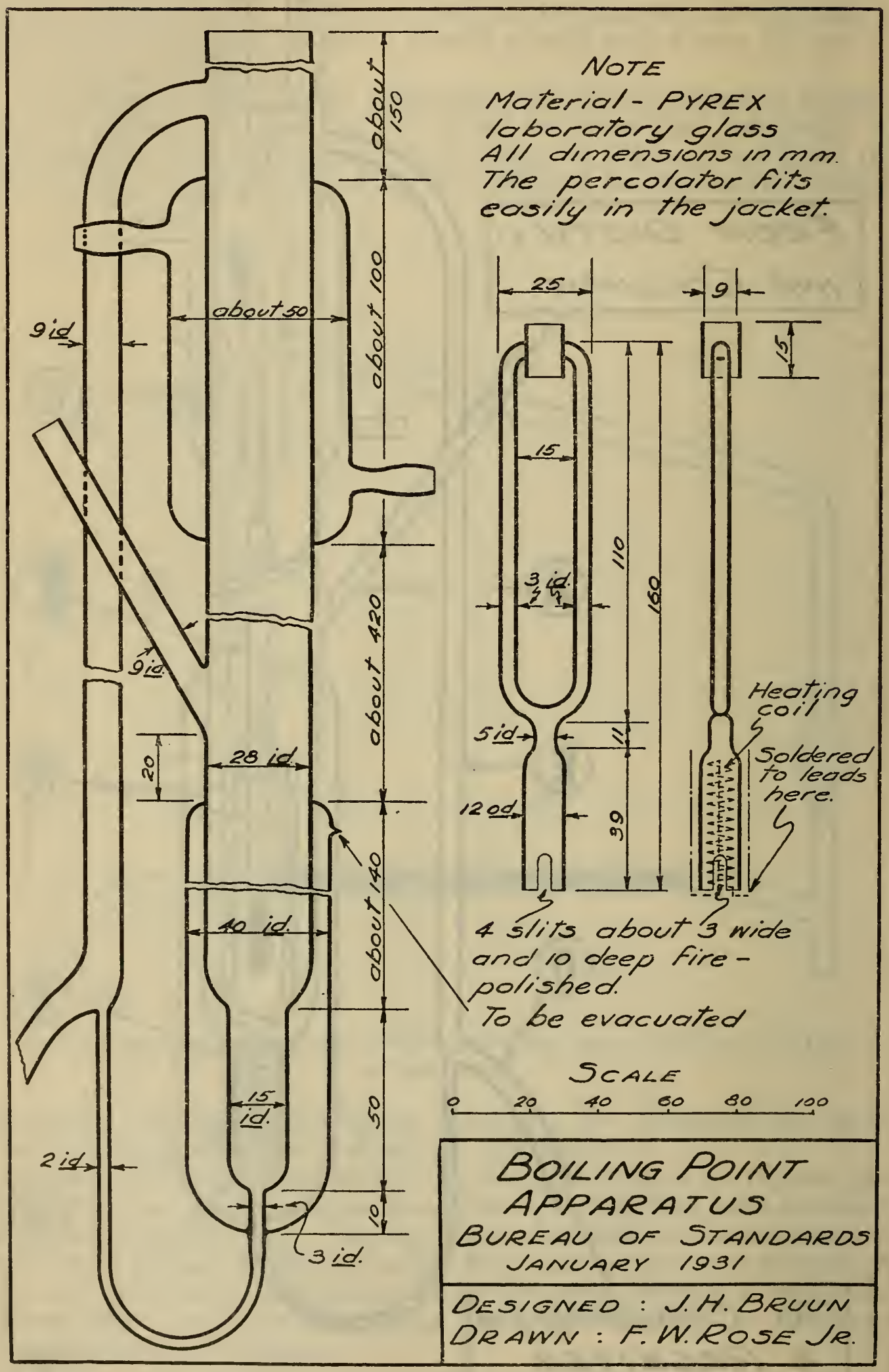

FIGURE 13.-Continuous boiling-point apparatus (working drawing) 


\section{CONDENSERS AND RECEIVER}

All the necessary specifications for the condensers and the receiver are given in Figure 14.

For vacuum distillations the vacuum system (for details see Appendix I) is connected to the still through $V$. In order to withdraw fractions from the receiver without breaking the vacuum in the still proper, the following procedure is used. The stopcocks $A$ and $B$ are closed and $\mathrm{CO}_{2}$ is admitted through $C$, after which the distillate is removed through $D$. The receiver is then reevacuated by means of an auxiliary pump through $C$ to the required pressure after which $C$ is closed and $A$ and $B$ are opened. During vacuum distillations leaks through the bore of $C$ and $D$ are prevented by placing these outlets in small beakers filled with mercury, or by attaching short pieces of rubber tubing, the lower ends of which are closed by glass rods. A receiver could be designed which did not necessitate the use of stopcocks, but it did not seem worth while for the present purpose to introduce this additional complication.

\section{MOUNTING OF THE STILL}

All parts of the rectifying still should be mounted on a rigid metal frame (for example, $4 \mathrm{~cm}$ (11/2 inch) angle iron) in order to prevent breakage caused by strains on the glass and to permit ready access to all parts of the assembly. Owing to the height of the rectifying column the mounting of these parts becomes a problem of the greatest importance. A very satisfactory method of mounting is shown in Figure 15. A supporting joint $A$, is used to connect the still pot and the column. The tapered section of $A$ is blown (in a carbon mold) to fit a tapered hole in the hard asbestos block $B$ which rests upon a second block $C$, cut in halves. These blocks, which are placed on a rigid metal support, carry the entire weight of the heavy still pot, the rectifying column, the reflux regulator, and the column jacket.

In mounting the still the following procedure is followed:

1. The block $B$ is placed around the neck of the still pot as shown, after which seal I between the still pot and the part $A$ is carefully made.

2. The entire still pot with the part $A$ as well as block $B$ is placed in an electric annealing oven and heated to $550^{\circ} \mathrm{C}$. for one hour. The oven is then allowed to cool to $450^{\circ} \mathrm{C}$., after which a temperature of $400^{\circ}$ to $450^{\circ} \mathrm{C}$. is maintained for about 12 hours.

3. Seal II is now made and carefully annealed on the bench. Sufficient length should be left between the column and seal II to avoid heating the first ring seal in the column.

4. The column jacket is then slipped over the rectifying column and the whole unit is placed in position upon the block $C$ which rests on a heavy metal support (indicated by a dash-dot line in fig. 15). At the top of the still no rigid support is desirable either for the rectifying column or for the column jacket, since they must be left free to expand when heated. To prevent lateral motion, an asbestoscovered ring or clamp is fastened loosely around the top of the column. For taller and heavier columns a counterweight system should be employed. (See Sec. XIII.)

Figure 16 is a photograph of five of these stills mounted on a single frame. 


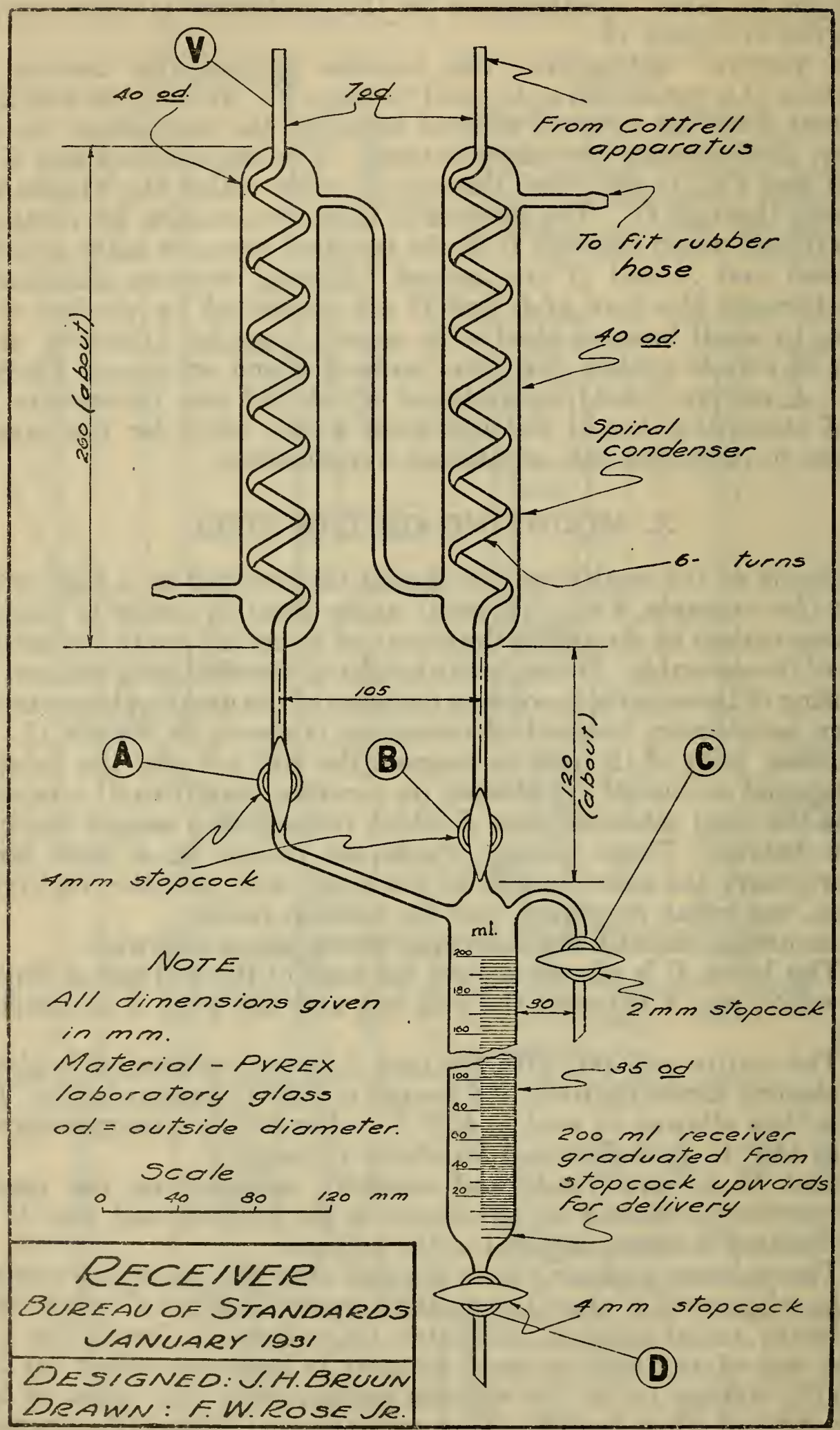

FIGURE 14.-Receiver and condensers 


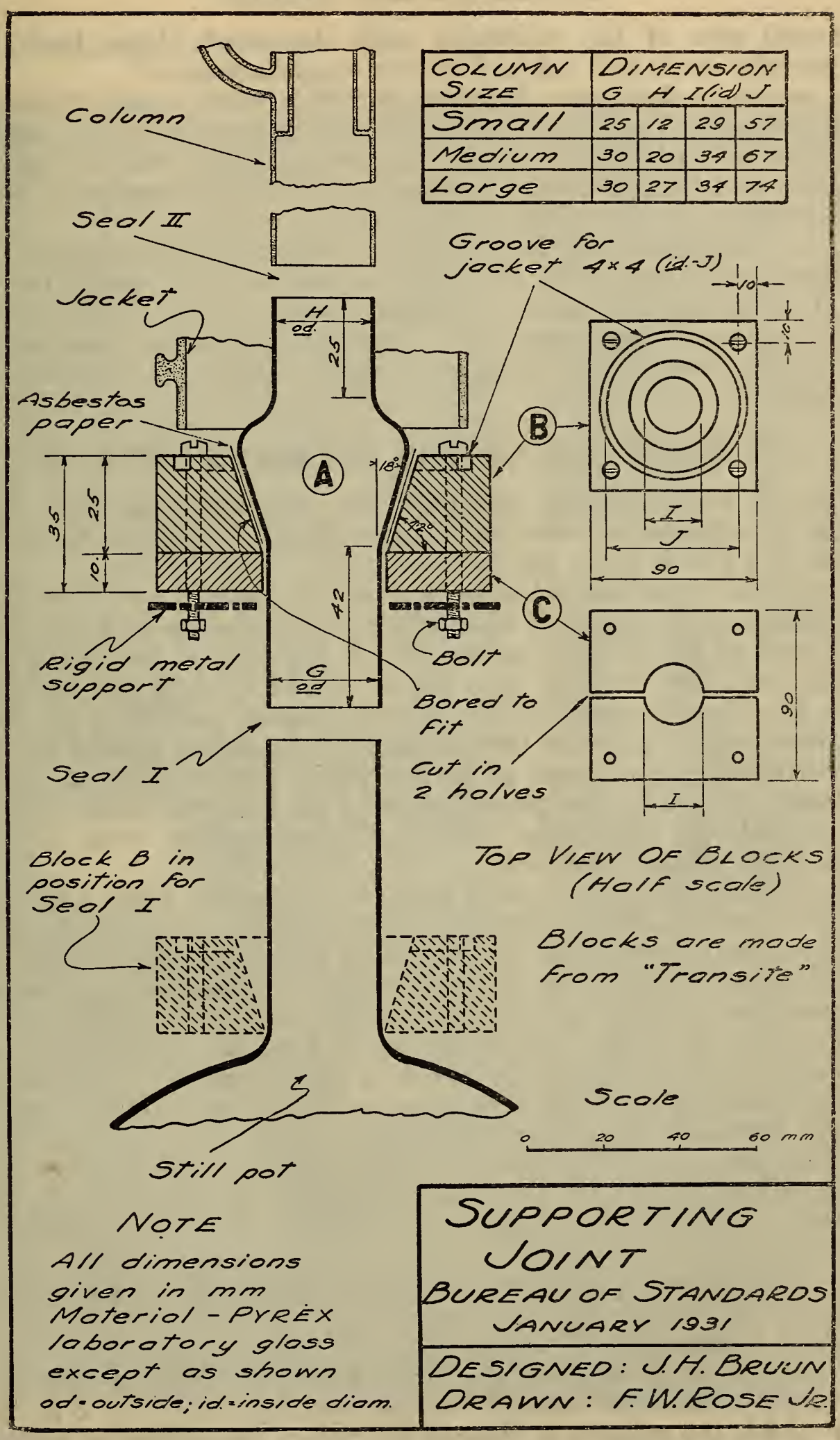

FIGURE 15.-Method used for supporting the column and the column jacket 


\section{MANUFACTURE AND TRANSPORTATION OF THE BUBBLING-CAP STILLS}

Several sets of the rectifying stills described above have been successfully made and shipped by two manufacturers. ${ }^{11}$

In order to facilitate shipping the stills were made up in nine different sections as follows: still pot with the gas inlet tube and the thermometer tube, filling tube, supporting joint and the asbestos support, rectifying column, column iacket, reflux regulator, boiling point apparatus, two spiral condensers, and receiver.

All of these parts can be shipped by express, if the necessary precautions are taken. For this purpose the column should be filled with lubricating oil in order to hold the loose bubbling caps in position and to prevent shattering them. The column is then packed in a box with sawdust, and inclosed within a larger box packed with excelsior.

\section{OPERATION OF THE BUBBLING-CAP STILL}

As soon as the cooling water on the condensers has been turned on and a slow stream of air has been admitted to the top of the column jacket, the still is ready for use.

The liquid to be distilled is introduced into the still pot, 1 (see fig. 1), through the filling tube, 4 , after which it is heated to its boiling point by means of the electric heater, 2. A low rate of boiling is maintained until the lower 5 to 8 plates of the column are operating. Then the electric current in the bottom heating section of the jacket is turned on. The other three heating sections are turned on when the corresponding sections of the rectifying column start to operate. In case any one of the plates shows a tendency to flood, the heat on the still pot should be turned off momentarily until the plate resumes its normal behavior.

As soon as the boiling-point apparatus becomes filled with distillate, its heating coil is turned on and the boiling point is determined. The four heating sections of the column jacket are then regulated so that the thermometer of the top section registers about the same temperature as that of the boiling-point apparatus. The bottom heating section of the jacket should be kept at a somewhat lower temperature than that of the liquid in the still pot. The two intermediate heating sections should be regulated to maintain approximately the same temperature gradient for the column jacket as the assumed tempreature gradient of the liquid on the different plates in the column. In this way nearly adiabatic conditions will prevail around the column and maximum efficiency in the distillation will be obtained.

If the distillation is carried out in an atmosphere of $\mathrm{CO}_{2}$, the gas is passed in through the gas inlet (for details see fig. 2) at a rate of about 20 bubbles per minute.

If the still is to be cleaned after a distillation, the residue in the still pot is sucked out through the thermometer tube (No. 5 in fig. 1) after which the column is cleaned by one of the following methods:

1. Most of the liquid in the column is drawn into the still pot by applying suction at the thermometer tube (No. 5 in fig. 1). The 11 Otto R. Greiner, 68 Marshall St., Newark, N. J.; E. Machlett and Sons, 50 William St., Long Island
City, N. Y. 
B S. Journal of Research, RP379

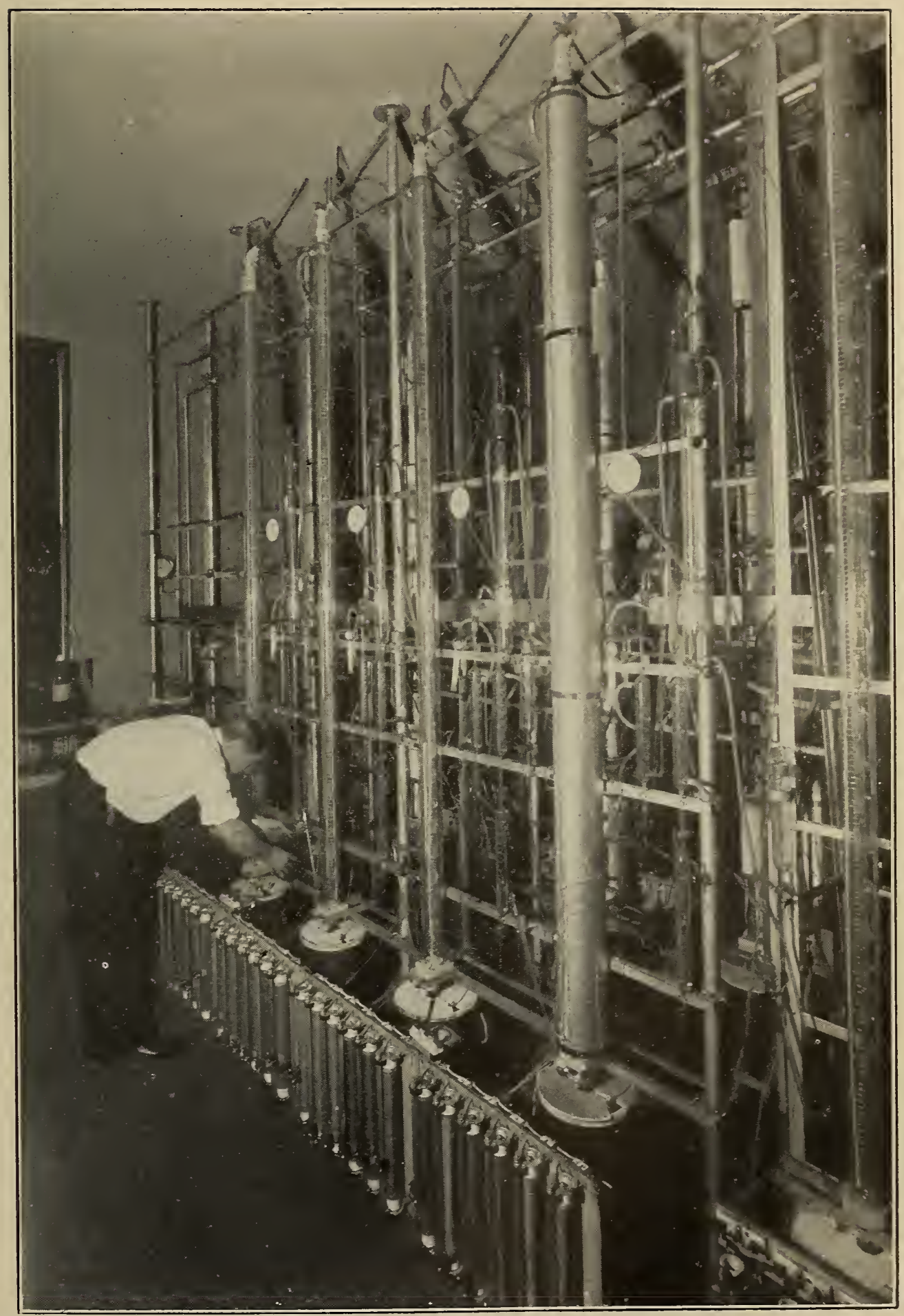

FIGURE 16.-Assembly of five 30-plate bubbling cap stills mounted on a single frame 
remaining liquid is distilled into the receiver by heating the column jacket and drawing air (or an inert gas) through the column by applying suction at the top of condenser, 14, Figure 1.

2. All of the liquid in the column is washed down into the still pot by means of a liquid poured into the thermometer tube at the top of the still. In the case of petroleum fractions, liquids such as water, methyl alcohol, or acetone may be used for this purpose, because they can afterwards be easily separated from the hydrocarbons. Water is, of course, separated by means of a separatory funnel, while acetone and methyl alcohol are removed from the hydrocarbons by repeated washing with water. ${ }^{12}$ The wash liquid is then removed from the column by method (1). By the use of the above methods a 30-plate column can be cleaned out and dried in about 20 to 30 minutes.

\section{COLUMNS FOR DISTILLATIONS AT PRESSURES LESS THAN $50 \mathrm{~mm}$}

For fractional distillations at low pressures, a packed column should be used. A laboratory still for vacuum distillation provided with a column packed with steel jack chain has been described elsewhere. ${ }^{13} 14$ If a high degree of separation is desired, a tall column should be employed. Figure 17 shows an all-glass rectifying still with an $11 \mathrm{~m}$ column packed with chromium-plated steel jack chain. When the height of a column reaches such a magnitude the combined weights of the packing, the column, and the column jacket will be considerable, and a special method of mounting becomes necessary. As shown in the figure a part (about 60 per cent) of this weight is carried by the counterweights, $W$, at the top of the still, so that no strains are introduced into the glass column or glass-column jacket when these expand or contract. The bottom support and other features of construction are similar to those of the bubble-cap stills already described.

Comparative runs with varying amounts of liquid in the column indicated that the efficiency of this still was greatest when there was flowing through the column a steady trickle of liquid, sufficient to wet the packing thoroughly. When operated just below the flooding point, the efficiency was materially decreased.

\section{STOPCOCK LUBRICANT}

The stopcocks throughout the distillation system should be lubricated, preferably with a lubricant which is insoluble or only slightly soluble in the liquids to be distilled. The selection of a suitable lubricant will therefore depend upon the physical and chemical nature of the distillate. Upon suggestion of T. P. Sager, of this bureau, polymerized diethylenephtalate and triethylenephtalate were tried as stopcock lubricant for distillation of petroleum fractions. While both of these compounds ${ }^{15}$ are practically insoluble in most hydro-

\footnotetext{
12 See J. H. Bruun and M. M. Hicks-Bruun, B. S. Jour. Research, vol. 5, p. 933; 1930.

13 J. H. Bruun, B. S. Jour. Research, vol. 2, p. 476; 1929.

14 This packing has been recommended by Dean, Hill, Smith, and Jacobs, Bur. of Mines Bull. 207, p. 9, 1922 , and has been used in several stills at the Bureau of Standards.

15 The preparation of these compounds has been described by W. H. Carothers and J. A. Arvin, J. Am. Chem. Soc., vol. 51, p. 2568, 1929, and by R. H. Kienle and A. G. Hovey, J. Chem. Soc., vol.51, p. 509, 1229 , and vol. 52, p. 3636, 1930.
} 
carbons they can easily be removed from the stopcocks by washing with acetone in which they are soluble. In order to obtain a lubricant of suitable consistency, we melted about 20 per cent of the polymerized triethylenephtalate with about 80 per cent of the polymerized diethylenephtalate. This mixture has been found to be a very satisfactory stopcock lubricant for distillation of petroleum fractions and hydrocarbons.

The stopcock on the reflux regulator shown in Figure 9 has been lubricated with a special lubricant, which has a softening point of $50^{\circ}$ to $60^{\circ} \mathrm{C}$. This lubricant was suggested by C. H. Binkley, of this bureau, and was prepared by heating equal mols of sebasic acid and ethylene glycol for about 20 hours at $180^{\circ} \mathrm{C}$. or about 15 hours at. $200^{\circ} \mathrm{C}$. The lubricant thus obtained is insoluble in aliphatic hydrocarbons, alcohol, and ether but is readily soluble in benzene, alkylhalides, and pyridine. For inorganic lubricants the reader is referred to recent papers by W. A. Boughton. ${ }^{16}$

\section{EFFICIENCY OF THE STILLS}

Many laboratory stills have been described in the literature in recent years. Distillations of various known mixtures have been carried out in these stills in order to illustrate their efficiencies. Unfortunately, however, a great many of these so-called efficiency tests give only the temperature-volume curve of the distillations as the criterion of efficiency. It is evident that an efficiency test based solely upon this curve is illusory and of little value, particularly if the components of the distilling mixture differ in boiling points by only a few degrees. The boiling range of a fraction may be a very unsatisfactory criterion for the composition of the distillates, because comparatively small differences in boiling points may correspond to large differences in composition. Moreover, the distillation temperatures, when measured in the usual manner, are influenced by many factors such as superheating of the vapor, rates of distillation, variations in the atmospheric pressure, etc.

During distillations at constant but very low rates, of mixtures consisting of substances which differ in boiling point by only a few degrees it is possible to obtain ordinary temperature readings at the still head which continuously indicate that the lower boiling constituent distills over at a concentration of about 100 per cent, while an accurate analysis might show that the distillate contains only 60 to 80 per cent of the lower boiling components. Extreme discrepancies like these are caused by the fact that the thermometer is not surrounded by vapor in sufficient quantities to register the true condensation temperature of the vapor mixture.

A more reliable method of determining the operating efficiency of a given still, which has been used by many investigators, is to analyze the distillates by some reliable method, and then to plot the composition-volume curve for a distillation under controlled conditions (i. e., with respect to reflux ratio, rate of distillation, etc.). The operating efficiencies of the rectifying columns described in this paper were determined by this method, using the following binary mixture. ${ }^{17}$ 


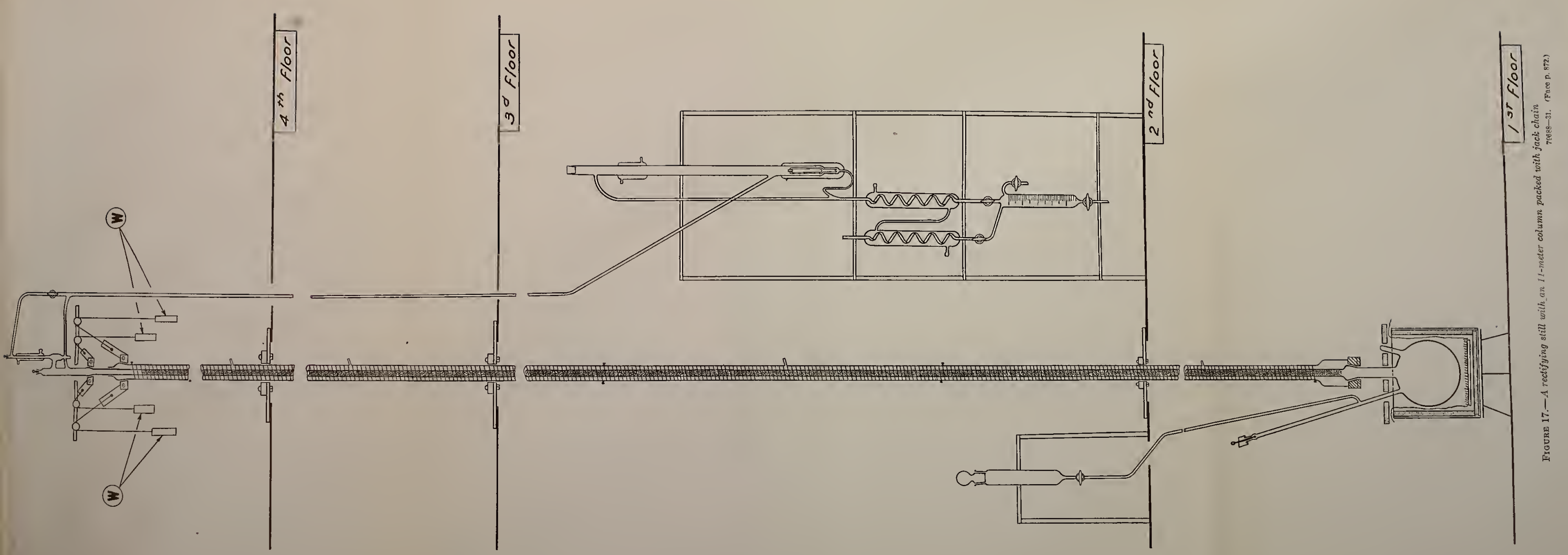


The initial charge as well as the distillates were analyzed by determining their refractive indices, after which the composition-volume curves for the distillations were plotted. These curves are shown in Figure 18. Curve I refers to a distillation in the 30-plate mediumsize column at $10: 1$ reflux ratio, which was maintained constant by means of the capillary reflux regulator shown in Figure 10. Curve $I t$ refers to the large-size column, which was operated at an unknown

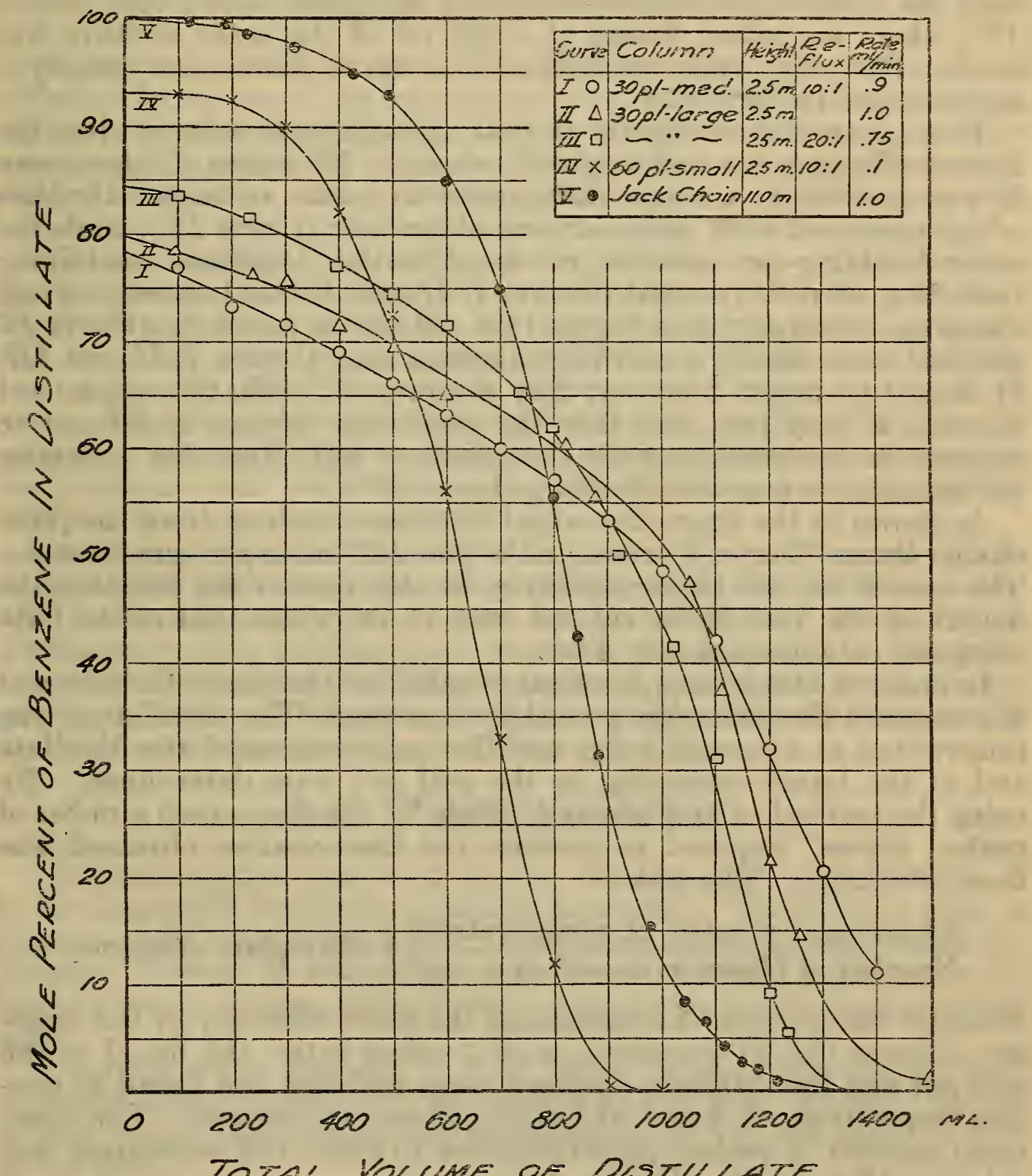

FIGURE 18.-Efficiency curves for the rectifying stills

Ordinate: Mole per cent of benzene in the distillate. Abscicca: Total voiume of distillate collected.

(probably 10:1 to 20:1) reflux ratio, maintained by means of the reflux regulator shown in Figure 9. Curve $I I I$ shows the results of a distillation in the same 30-plate large-size column, but the distillation was in this case carried out at a reflux ratio of $20: 1$. Curve $I V$ is the composition-volume curve for a distillation in the 60-plate small size still which was operated at a reflux ratio of $10: 1$. The heights of all these columns were about $2.5 \mathrm{~m}$. 
For comparison, a distillation was carried out in the jack-chain packed-column still (shown in fig. 17), the column of which was $11 \mathrm{~m}$ high (or 4.5 times as high as the bubbling-cap stills.) This distillation, which was carried out at a reflux ratio of about $15: 1$, gave Curve $V$, Figure 18. This is also reproduced in Figures 23 and 24 .

The initial charge for these distillations was in every case 1,500 $\mathrm{ml}$ of a 50:50 mole per cent mixture of benzene and ethylene chloride, with the exception of the distillation in the small column still (Curve IV), where an initial charge of $1,000 \mathrm{ml}$ of the same mixture was used. The reflux ratios as well as the rate of distillation employed are recorded in Figure 18.

It is apparent from Figure 18 that the small-size column gives the highest efficiency per unit height of column. By means of this column it was possible in a single distillation to obtain an initial distillate which consisted of 93 mole per cent of benzene (Curve $I V$ ), while the other bubbling-cap columns produced initial distillates containing from 78 to 84 mole per cent (Curves $I, I I$, and $I I I$ ) of benzene. From the shape of the curves in Figure 18 it will also be noted that Curve $I V$ declines more rapidly toward the abscissa than Curves $I, I I$, and $I I I$. It should be noted, however, that the rate of distillation $(1 / 10 \mathrm{ml}$ per minute) is very low, and that the small-size column is difficult to operate in comparison with the medium and large-size columns, particularly for fractions distilling above $100^{\circ} \mathrm{C}$.

As shown in the figure the initial distillate obtained from the jackchain column (Curve $V$ ) consisted of about 99 mole per cent benzene. The reason for the higher efficiency in this case is the fact that the height of the jack-chain column was $11 \mathrm{~m}$, while that of the bubbling-cap columns was only $2.5 \mathrm{~m}$.

In order to obtain an approximate value for the plate efficiencies of the columns the following procedure was used: The distillation was interrupted at a certain point and the compositions of the distillate and of the liquid remaining in the still pot were determined. By using the method of McCabe and Thiele, ${ }^{18}$ " the theoretical number of perfect plates" required to produce the fractionation obtained was then calculated. The ratio:

$$
\text { Theoretical number of perfect plates } \times 100=\text { plate efficiency }
$$

Thus for the purpose of determining the plate efficiency of the largesize column (fig. 5) (operated at 20:1 reflux ratio) the liquid in the still pot and the distillate obtained were analyzed and found to contain respectively 43.5 and 80.2 mole per cent of benzene. The minimum number of perfect plates required to effect this separation was calculated ${ }^{18}$ to be 26 . Hence:

$$
\frac{26}{30} \times 100=87 \text { per cent plate efficiency }
$$

which represents a very satisfactory efficiency for a laboratory column.

Because of the difficulties involved in maintaining perfectly adiabatic conditions around a rectifying column of small diameter, it is probable that even if a constant reflux ratio $(20: 1)$ at the top of the

18 W. L. McCabe and E. W. Thiele, Ind. Eng. Chem., vol. 17, p. 605; 1925. 
column is maintained, the reflux may be somewhat increased in the lower parts of the column. This increase is due to the condensation which results from the loss of heat from the column to the surrounding column jacket. In view of this slight uncertainty in the reflux ratio the calculated plate efficiency should be considered only as an approximate value.

\section{SELECTION OF A LABORATORY STILL}

As pointed out in Section XV, a higher degree of separation is obtained by means of the bubbling-cap stills than with the jack-chain stills provided that rectifying columns of equal height and diameter are compared under the same conditions with respect to reflux ratio, rate of distillation, etc. However, the cost of a bubbling-cap still will be nearly twice that of a jack-chain still of the same height.

Whether the higher efficiency of the bubbling-cap column justifies the additional expense of installation will depend upon the particular distillation problem involved. For instance, in laboratories where a still is needed only for occasional distillation it would seem that the less expensive jack-chain still is entirely satisfactory, provided, of course, that the packing is not attacked by the distilling liquids. If sufficient vertical height is available a tall jack-chain column will be very efficient and has the advantage that it may be employed for distillation at very low pressures.

On the other hand, if the available vertical space is limited by the height of the laboratory room, the bubbling-cap column will be preferable for distillations which require the highest degree of separation. Furthermore, the bubbling-cap column is recommended for laboratories where continuous distillations are to be carried out during longer periods of time. The saving in time in such a case will fully justify the additional expense of the bubbling-cap still.

\section{ACKNOWLEDGMENT}

This investigation has been carried out as a part of project No. 6 of the American Petroleum Institute Research. The authors wish to express their thanks to $\mathrm{E}$. W. Washburn, chief of the chemistry division, Bureau of Standards, and director of this project, for his kind interest, advice, and criticism during the development of this work.

The authors are indebted to F. W. Rose, jr., for his preparation of all of the figures included in this paper, and to E. O. Sperling for his helpful suggestions and skillful glassblowing during the development of the stills.

\section{APPENDIX 1}

\section{REGULATION OF THE PRESSURE WITHIN THE STILL ASSEMBLY}

In order to maintain a constant pressure during the distillations, the arrangement shown in Figure 19 is used. The rectifying stills indicated by the small circles, $S$, are connected directly to the main pressure line and to a welded 350 -liter steel tank, $B$, which prevents

$79688-31-8$ 
sudden changes in pressure. The main pressure line is connected through a cooling trap, $T$, to a vacuum pump, $P$, the manostat, $N$, and the manometer, $M$.

The manostat is connected to the relay, $R$, which starts the pump, $P$, when the pressure increases about $0.05 \mathrm{~mm}$ in the still assembly. An inclined butyl phthalate manometer (not shown in the figure) is used as a pressure gage. This is designed so as to restrict the total pressure change to the sloping end of the manometer. A movement of $0.6 \mathrm{~mm}$ of the meniscus corresponds to a pressure change of $0.01 \mathrm{~mm}$ mercury within the system.

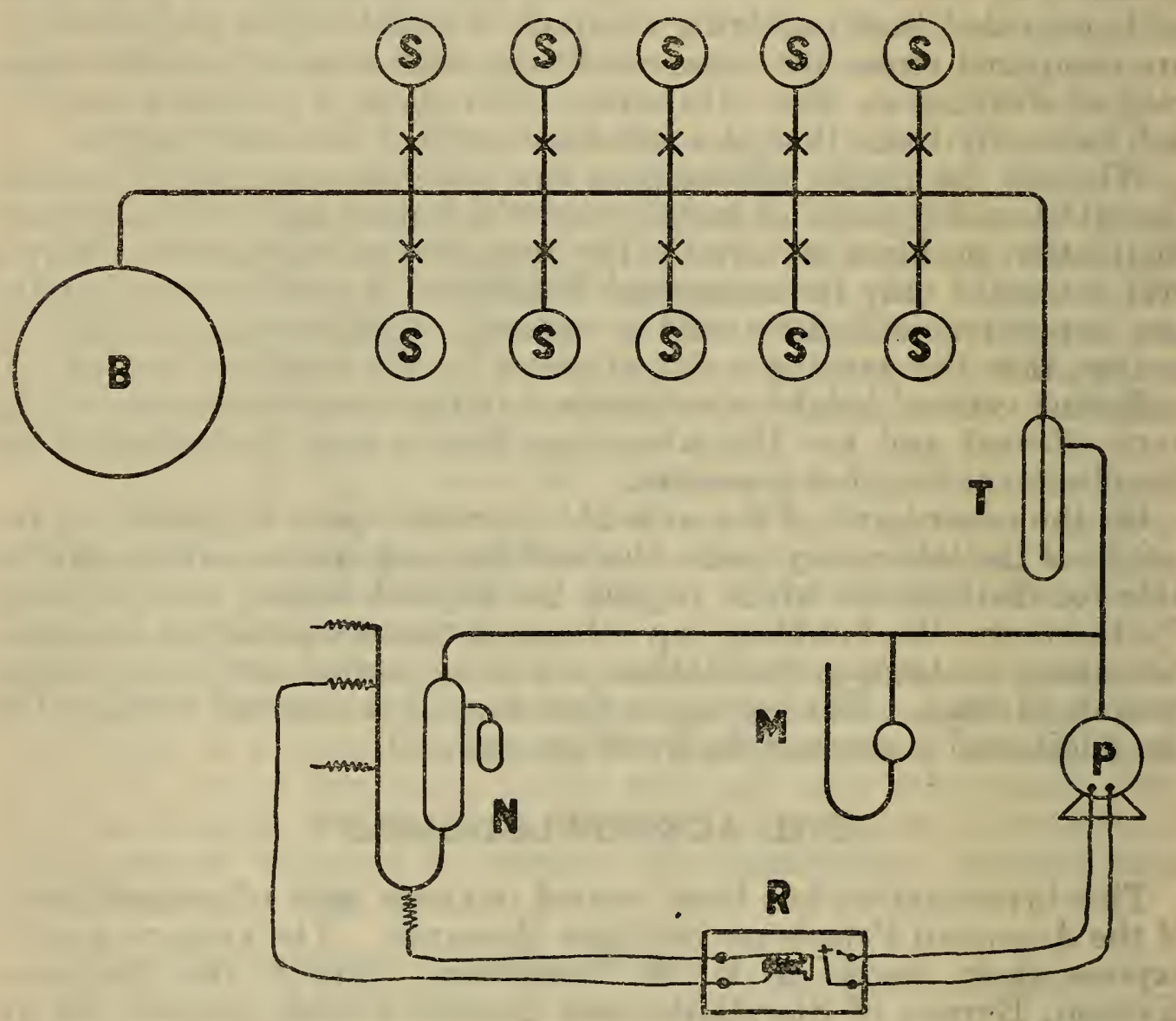

FIGURE 19.-Diagram showing the method used for regulation of the pressure within the still assembly

Figure 20 is a working drawing of the manostat. ${ }^{19}$ The tungsten lead, 1 , is permanently connected to one terminal of the relay. (See fig. 19.) The other relay terminal is connected to the tungsten lead, 2 , 3 , or 4 , depending upon the desired pressure. The working pressure range for the manostat in Figure 20 is from 25 to $215 \mathrm{~mm}$. The three contact points, 2,3 , and 4 , are for pressures of 100,150 , and $215 \mathrm{~mm}$, respectively. Intermediate pressures can be obtained by increasing or decreasing the level of mercury in bulb, $U$, by tilting the apparatus to adjust the amount of mercury in the auxiliary bulb, $V$. More refined adjustment is obtained by having the manostat so mounted that by tilting it sideways the mercury levels can be changed

${ }_{10}$ This is a modification of a pressure regulator described by H. L. Cox, Ind. Eng. Chem., Anal. Ed. , vol. 1, p. $7 ; 1929$. 


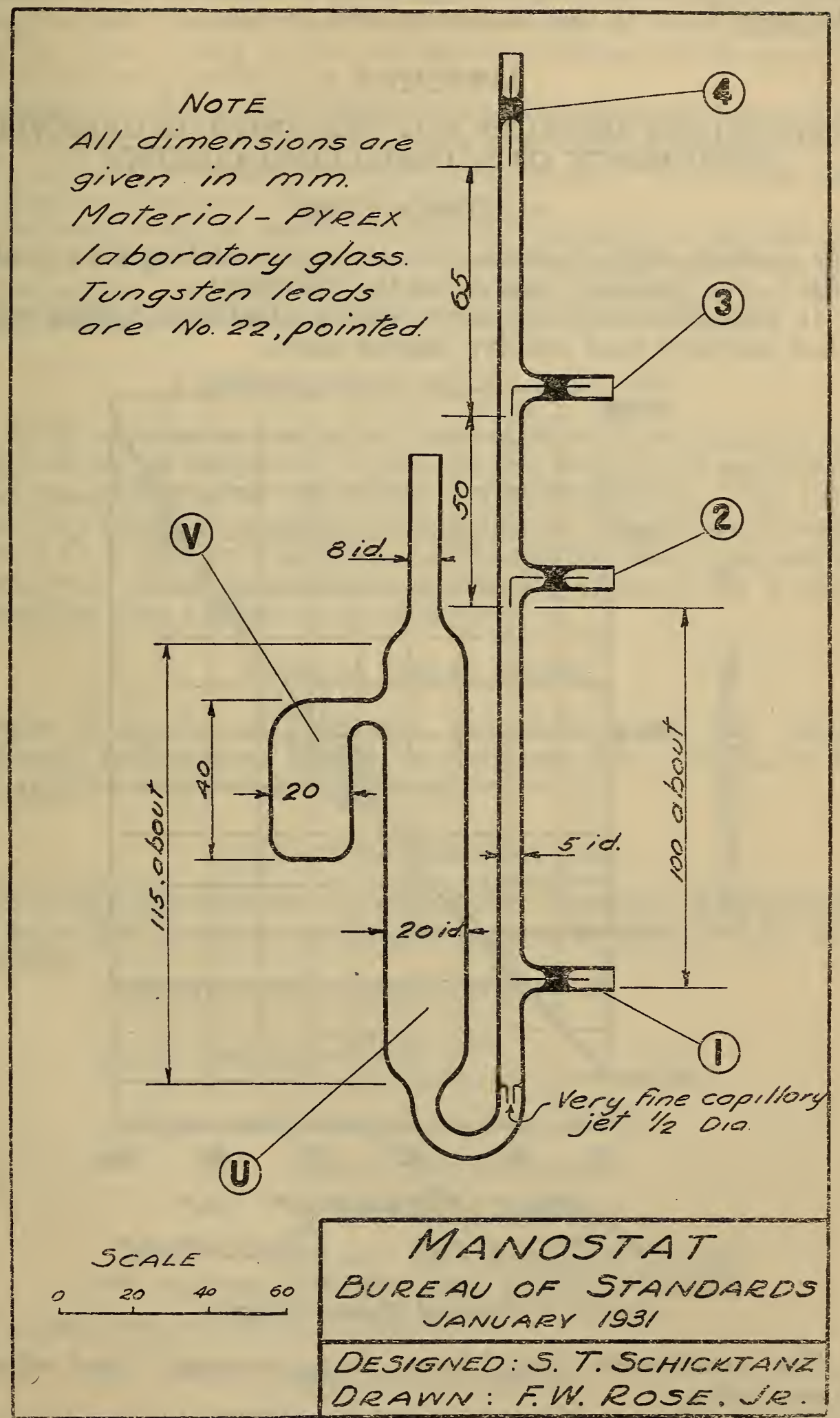

Figure 20.-The manostat 
by a small fraction of a millimeter. With this arrangement the pressure within the system can be maintained constant to about $0.05 \mathrm{~mm}$ in the range from $25 \mathrm{~mm}$ to atmospheric pressure.

\section{APPENDIX 2}

\section{A CONVENIENT MIXTURE FOR TESTING THE OPERATING EFFICIENCY OF A DISTILLING COLUMN ${ }^{20}$}

\section{INTRODUCTION}

The desirable characteristics of a mixture for testing the operating efficiency of a distilling column are the following:

1. It should be composed of two stable substances boiling only a few but not more than say five degrees apart.

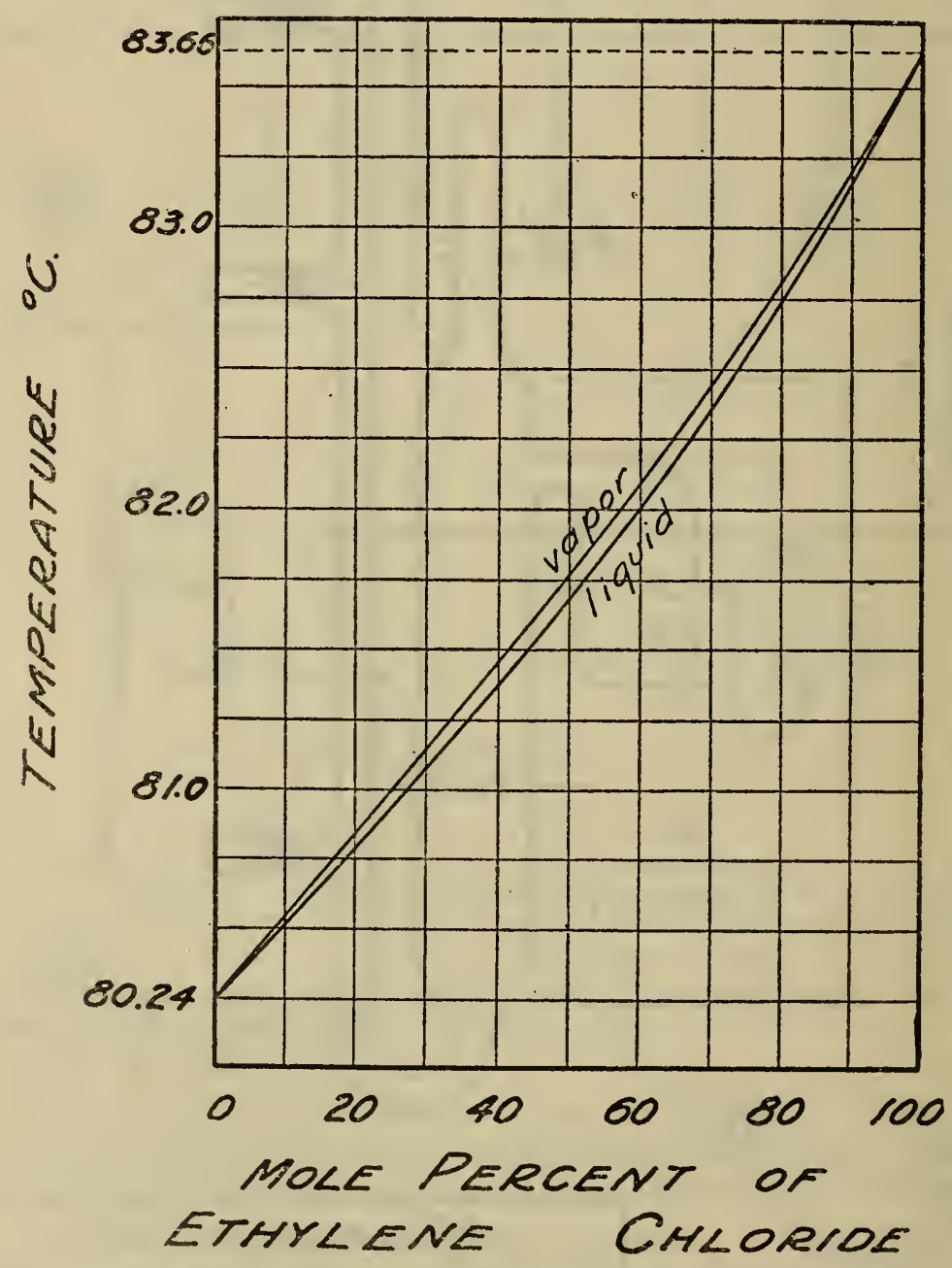

FIGURE 21.-Bouling point-composition curve for mixtures of benzene and ethylene dichloride

2. The constituents should form an approximately ideal solution with each other.

3. The constituents should be readily obtainable.

4. A convenient method for the rapid and accurate analysis of the mixture should be available.

\footnotetext{
20 Issued originally as Bureau o Standards Letter Circular No. 289; October, 1930.
} 
A mixture of $\mathrm{CCl}_{4}$ and $\mathrm{C}_{6} \mathrm{H}_{6}$ meets all of the above requirements except No. 2. Failure to meet this requirement means that fractionation near one end of the composition range is more difficult than near the other, owing to the fact that the boiling point-composition curves are not symmetrical.

A better mixture can be obtained by substituting ethylene chloride, $\mathrm{C}_{2} \mathrm{H}_{4} \mathrm{Cl}_{2}$, for $\mathrm{CCl}_{4}$. This liquid forms a practically ideal solution with $\mathrm{C}_{6} \mathrm{H}_{8}$ and also meets all of the other requirements. The boiling pointcomposition diagram is shown in Figure 21.

The mixture can be easily and rapidly analyzed by measuring the refractive index. The density would serve equally well as an index of composition but would require a careful determination of the density-composition relation, which is not known at present.

\section{PREPARATION OF THE MIXTURE}

A 50-50 mole per cent mixture ( 1 part $\mathrm{C}_{6} \mathrm{H}_{6}$ to 1.2683 parts $\mathrm{C}_{2} \mathrm{H}_{4} \mathrm{Cl}_{2}$, weights in air) of the pure dry liquids should be prepared and kept in a bottle containing some "Dehydrite" $\mathrm{Mg}\left(\mathrm{ClO}_{4}\right)_{2} \cdot 3 \mathrm{H}_{2} \mathrm{O}$. This drying agent has a negligible solubility in the mixture. The refractive indices at $25.2^{\circ} \mathrm{C}$. of each pure liquid and of the mixture should be carefully determined. This temperature is selected because the available refractivity data happen to be for $25.2^{\circ} \mathrm{C}$.

\section{USING THE MIXTURE}

Before filling the still pot with the mixture, the pot, column, condenser, and receiver should be clean and dry. Drying is best accomplished with a stream of dry air.

\section{ANALYZING THE FRACTIONS}

The distillation fractions are analyzed by measuring their refractive indices at $25.2^{\circ} \mathrm{C}$. and computing the composition with the aid of the equations

$$
\begin{aligned}
(M \text { per cent })_{E} & =(M \text { per cent })_{\text {calc. }}-\Delta \\
(M \text { per cent })_{\text {calc. }} & =\frac{100\left(n_{B}-n\right)}{n_{B}-n_{E}} \\
(M \text { per cent })_{E} & =\text { mole per cent } \mathrm{C}_{2} \mathrm{H}_{4} \mathrm{Cl}_{2} \\
n_{B} & =n_{D}^{25.2^{\circ}} \text { for the benzene } \\
n & =n_{D}^{252^{\circ}} \text { for the fraction } \\
n_{E} & =n_{D}^{25.2^{\circ}} \text { for the ethylene chloride }
\end{aligned}
$$

$\Delta$ is read from the curve in Figure 22.

\section{EFFICIENCY CURVE}

The efficiency graph for a given still and a given mode of operation is obtained by plotting volume of fraction against composition as illustrated in Figure 23 or by making the corresponding differential plot as illustrated in Figure 24. 
After completing the test the fractions may be returned to the stock bottle, the contents of which can be kept at the 50-50 composition by "doctoring" occasionally with a little of the required pure component until the original refractive index is restored.
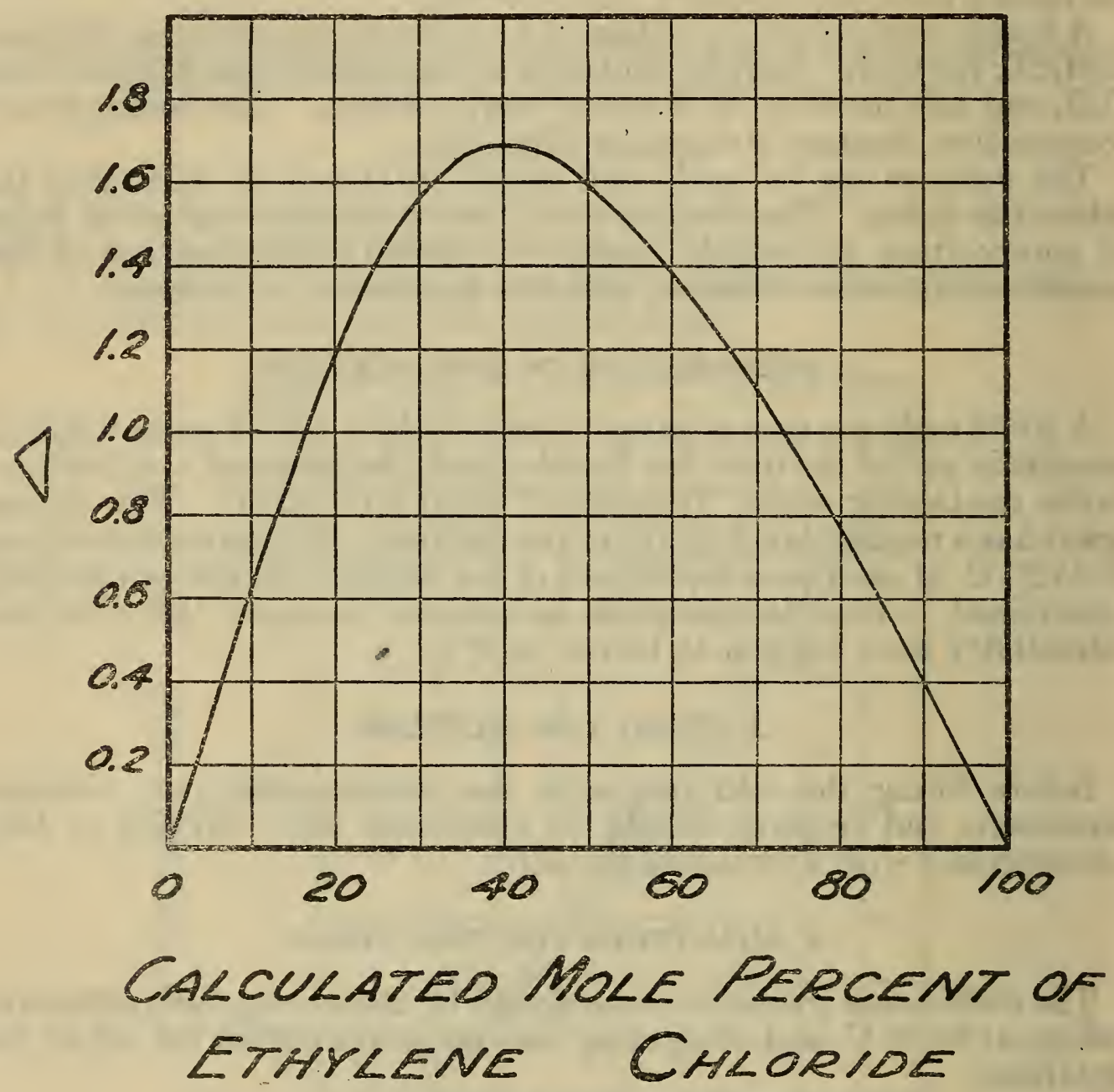

FIGURE 22.-Correction curve for the refractive index

\section{PURIFICATION OF THE CONSTITUENTS}

(a) ETHYLENE CHLORIDE

Ethylene chloride, equal in quality to the best grade made by Eastman, required to be distilled only once from a suitable drying agent (Dehydrite) in a good column still with rejection of the initial and final fractions.

Yield, about 85 volume per cent.

$n^{25.2^{\circ}}$ is 1.4418 .

Freezing range: $34.9^{\circ}$ to $35.1^{\circ} \mathrm{C}$.

(b) BENZENE

A good grade of C. P. benzene should be selected and treated as follows:

(1) Wash for 1 hour (preferably in a shaking machine) with 0.1 of its volume of $\mathrm{C}$. $\mathrm{P}$. concentrated $\mathrm{H}_{2} \mathrm{SO}_{4}$.

(2) Wash successively with water, dilute $\mathrm{NaOH}$ and water. 


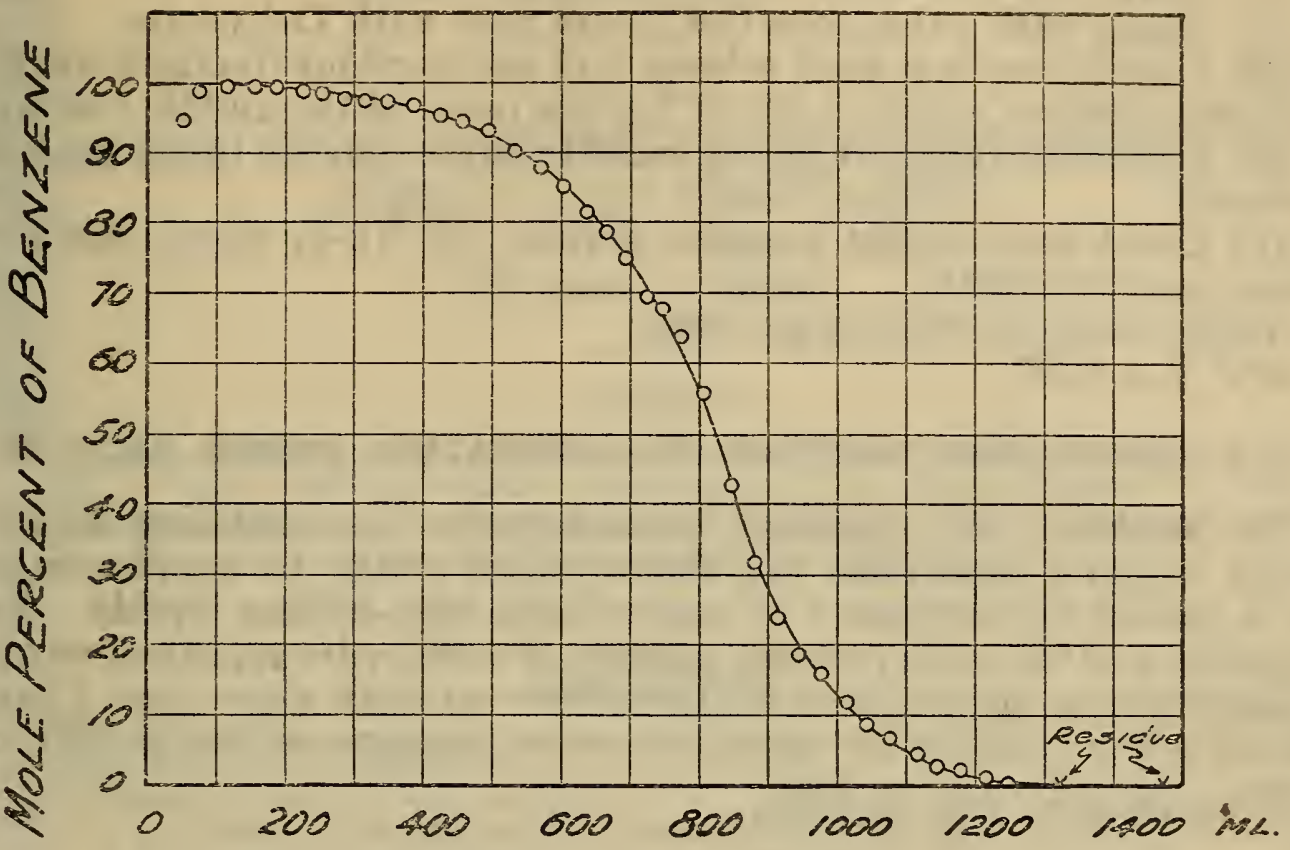

TOTAL VOL UME OF DISTILLATE

FIGURE 23.-Efficiency test of an 11-meter packed column

Ordinate: Mol per cent of benzene in distillate. Abscissa: Total volume of distillate collected.

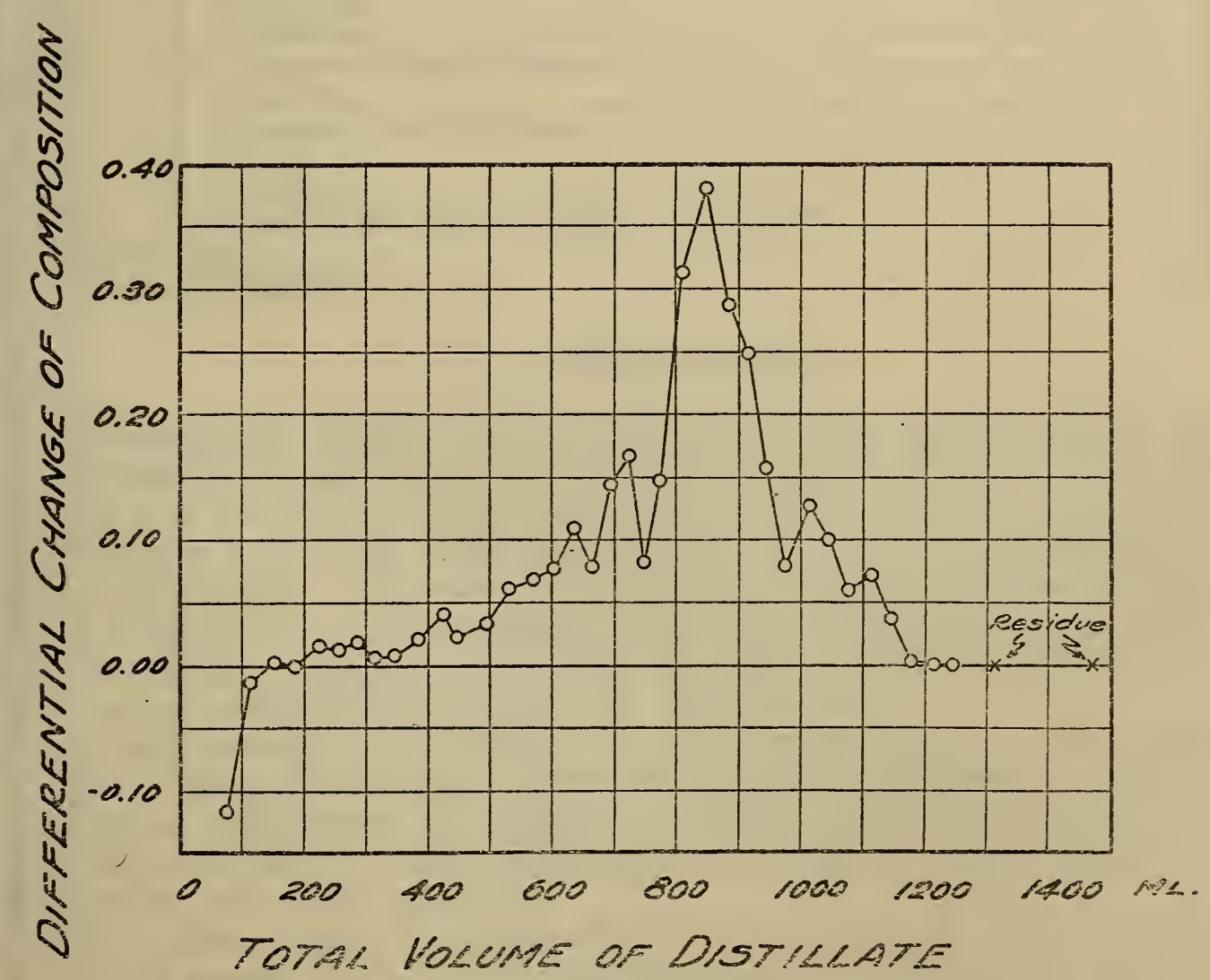

FIgdRE 24.-Differential graph of Figure 23 
(3) Shake with mercury until tarnishing ceases.

(4) Shake with anhydrous $\mathrm{CaCl}_{2}$ and then with Dehydrite.

(5) Distill once in a good column still and combine fractions having refractive indices within $\pm 5 \times 10^{-4}$ of the index of the middle fraction.

(6) Crystallize once, rejecting mother liquor (about 15 per cent by volume).

(7) Distill from bright metallic sodium (or $\mathrm{Na}-\mathrm{K}$ alloy) and preserve over Dehydrite.

Yield, about 65 volume per cent.

$n_{D}^{25.2^{\circ}}$ is 1.4976 .

\section{A CONVENIENT MIXTURE FOR OPERATION UNDER VACUUM}

For studying the operating characteristics of a distilling column under vacuum conditions the above mixture may be supplemented by a second one composed of two suitable high-boiling liquids. For example a 50:50 mole per cent mixture of tetrahydronaphthalene and $n$-amylbenzene should meet the conditions set forth above (sec. 1) and should prove suitable for operation under pressures as low as $10 \mathrm{~mm}$.

Washington, July 8, 1931. 